\title{
Scaling behavior of tethered crumpled manifolds with inner dimension close to $D=2$ : Resumming the perturbation theory.
}

\author{
Henryk A. Pinnow ${ }^{1}$ and Kay Jörg Wiese ${ }^{1,2 *}$ \\ ${ }^{1}$ Fachbereich Physik, Universität Essen, 45117 Essen, Germany \\ ${ }^{2}$ Laboratoire de Physique Théorique, Ecole Normale Supérieure, 24 rue Lhomond, 75005 Paris, France
}

\begin{abstract}
The field theory of self-avoiding tethered membranes still poses major challenges. In this article, we report progress on the toy-model of a manifold repelled by a single point. Our approach allows to sum the perturbation expansion in the strength $g_{0}$ of the interaction exactly in the limit of internal dimension $D \rightarrow 2$, yielding an analytic solution for the strong-coupling limit. This analytic solution is the starting point for an expansion in $2-D$, which aims at connecting to the well studied case of polymers $(D=1)$. We give results to fourth order in $2-D$, where the dependence on $g_{0}$ is again summed exactly. As an application, we discuss plaquette density functions, and propose a Monte-Carlo experiment to test our results. These methods should also allow to shed light on the more complex problem of self-avoiding manifolds.
\end{abstract}

Keywords: polymer, polymerized membrane, renormalization group, exact resummation.

Submitted to Nuclear Physics B

*Email: hpinnow@ sinits.com, wiese@lpt.ens.fr 


\section{Contents}

1 Introduction $\quad 3$

2 Model and physical observables $\quad 5$

2.1 The model . . . . . . . . . . . . . . . . . . . . . 5

2.2 Plaquettes-density correlation functions . . . . . . . . . . . . . 8

2.3 Delocalization transition . . . . . . . . . . . . . . . 10

3 Complete summation of the perturbation series $\quad 10$

3.1 Perturbation theory . . . . . . . . . . . . . . . . 10

3.2 Complete summation in fixed internal space dimension $D=2 \ldots \ldots$. . . . 12

3.3 Asymptotic scaling behavior . . . . . . . . . . . . . . . . 12

4 Crossover to polymers 16

$4.1 \quad(2-D)$-expansion on the torus $\ldots \ldots \ldots \ldots \ldots$

4.2 Resummed contributions to the expansion in $2-D$ up to fourth order . . . . 20

4.3 Renormalized coupling . . . . . . . . . . . . . . . . . . . . . 24

4.4 Guessing the exact $\tilde{g}(r) \ldots \ldots \ldots \ldots \ldots$

5 Conclusion $\quad 27$

$\begin{array}{llr}\text { A The propagator } & 28\end{array}$

B Calculation of the diagrams in the $(2-D)$-expansion $\quad 29$

$\begin{array}{lll}\text { R References } & 33\end{array}$ 


\section{Introduction}

One major problem in statistical physics is the effect of interactions on the thermodynamical properties of extended fluctuating geometric objects. In general, multi-particle attractive or repulsive interactions are involved. One may divide these into two classes: Either (i) one may study the interaction of a single fluctuating object with itself as for instance the well known excluded volume interaction between any two monomers in a long polymer chain in a good solvent. There, the interaction leads to universal long-distance properties of chains as for example the anomalous scaling of the mean squared end-to-end distance. Or (ii), the interaction may act between different manifolds or between a single manifold and a fixed non-fluctuating object. It is then interesting to study how thermal fluctuations affect the depinning of the manifold from an attractive substrate as well as the steric repulsions from a wall. Finally, both cases can appear together.

Whatever the situation is, it is usually well understood as long as the fluctuating objects are onedimensional [1-4]. Referring to the example mentioned above, the long-distance properties of self-avoiding polymers can be analyzed with renormalization group techniques [5-7], either in the continuous Edwards Hamiltonian [8],

$$
\mathcal{H}[\vec{r}]=\frac{1}{2} \int_{x \in \mathcal{M}}(\nabla \vec{r}(x))^{2}+\frac{b_{0}}{2} \int_{x \in \mathcal{M}} \int_{y \in \mathcal{M}} \delta^{d}(\vec{r}(x)-\vec{r}(y)),
$$

or by mapping this model on a local $O(N)$ symmetric $\varphi^{4}$-theory in the limit of $N=0$ components $[9,3,1]$. The critical exponents describing the long-distance properties are related to the critical exponents of the corresponding $N$-vector model at the critical point. What makes (1.1) a non-standard theory is that the interaction is non-local, and not a polynomial of the field.

Obtaining the corresponding results for membranes poses considerable challenges. The generalization of polymers to $2 D$-surfaces are crystalline fixed-connectivity membranes as they appear for instance in the spectrin network of cell membranes. Considering "phantom" membranes which can freely fold into itself, the existence of a bending rigidity induced phase transition separating a high rigidity, low temperature flat phase from a low rigidity, high temperature crumpled phase is well established [10-15]. This is in contrast to polymers, which are always crumpled on large scales. The scaling properties of the crumpled phase of phantom membranes are described by the $2 D$ generalization of the free field part in (1.1). Taking self-avoidance into account, which is modeled in (1.1) through the short-range two-body interaction, we expect more swollen manifolds than those predicted by the free theory, which will be expressed in a non-trivial radius of gyration exponent $\nu$ :

$$
R_{g} \sim L^{\nu}, \quad 0 \leq \nu \leq 1
$$

where $L$ denotes the linear internal size of the membrane, and the radius of gyration $R_{g}$ is obtained from the effective extend of the membrane in external space. In the case of polymers $R_{g}$ scales like the end-to-end distance. Much effort has been spent on calculating corrections to the radius of gyration exponent within an expansion in the deviation $\varepsilon$ from the critical space dimension $[16,17]$. These calculations can not be performed directly for membrane-dimension $D=2$, since the naive scaling dimension of the coupling in (1.1) equals

$$
\varepsilon(D, d):=\left[b_{0}\right]=2 D-\frac{2-D}{2} d,
$$


where $d$ denotes the dimension of the embedding space, such that $\epsilon$ is always non-zero as $D \rightarrow 2$, for any embedding dimension $d$. Equivalently, the critical embedding dimension defined through $\varepsilon\left(D, d_{c}(D)\right)=0$ becomes infinity in this limit. The reason is that the nonself-avoiding membrane densely fills out the embedding space, such that it always "sees" the interaction. A way to circumvent this problem is to set up the expansion about any point $(D<$ $\left.2, d_{c}(D)\right)$ and to extrapolate along an appropriate path in the $(D, d)$-plane to the physically interesting point $(D, d)=(2,3)$ [18-24]. To second order in $\varepsilon$ one then finds a radius of gyration exponent of $\nu \approx 0.86[16,17]$, which is a strong correction with respect to the only logarithmic dependence in the non-interacting theory, and indicates the existence of a crumpled phase, for which $\nu \geq 2 / 3$ follows from the fact that a membrane has a finite volume.

However, there is no evidence for a crumpled phase in experiments [25-28]. Latest Monte-Carlo simulations on plaquette-models [29-32] starting from a discretization of the $2 D$ generalized Hamiltonian (1.1) with system sizes of up to $\approx 17000$ plaquettes shows considerable evidence for a vanishing of the above mentioned crumpling transition in the presence of self-avoidance, such that even on large scales fixed-connectivity membranes stay always flat with a radius of gyration exponent of $\nu \approx 1$. The final purpose of this work is to develop techniques which allow to go beyond the two-loop result. So far, we developed such techniques for a simplified model, which reduces the non-local self-avoiding interaction in (1.1) to self-avoidance with only a single point, e.g. the origin $o$ in the membrane:

$$
\mathcal{H}[\vec{r}]=\frac{1}{2} \int_{x \in \mathcal{M}}(\nabla \vec{r}(x))^{2}+g_{0} \int_{x \in \mathcal{M}} \delta^{d}(\vec{r}(x)-\vec{r}(o)) .
$$

This is a special case of a more general model of the interaction of a phantom tethered manifold with a single point in embedding space, which is related to case (ii). The corresponding physical situation to think of is the binding and unbinding of a long chain as e.g. a polymer or a membrane from a wall or the wetting of an interface. More precisely, we study the interaction of a single freely fluctuating manifold with another non-fluctuating, fixed object. Depending on whether the interaction is attractive or repulsive, one can distinguish two different scenarios: One may either observe a delocalization transition from an attractive substrate as in wetting phenomena or steric repulsions by a fluctuating manifold. Both cases have in common that excluded volume effects become important. These scenarios have already been discussed in [33]. The result of [33] is the complete resummation of the perturbation series for the effective coupling in the case of $2 D$-membranes. The long-distance behavior of the resummed theory turned out to be non-trivial in the sense that it emerged from the limiting behavior of a scale invariant theory resulting in an effective coupling growing logarithmically instead of approaching some finite fixed-point value as one would expect it. This, together with the extremely slow convergence of the perturbation series makes the analysis of the fully resummed theory a must, because all finite loop calculations fail to extract the correct large distance properties. The importance of the result becomes evident as soon as one compares it with extrapolations obtained from the $\varepsilon$-expansion at the 2-loop level [33]. Besides the necessity of calculating diagrams numerically with considerably raising effort as the loop order becomes higher, the $\varepsilon$-expansion has turned out not to be able to make reliable predictions for $D \lesssim 2$. This problem persisted, though we exploited the freedom to set up the expansion about any point $\left(D<2, d_{c}(D)\right), d_{c}(D)=\frac{2 D}{2-D}$ being the critical embedding dimension for given internal dimension $D$ and to expand both in $D$ 
and $d$ along any appropriate extrapolation path to some physically interesting point $(D=2, d)$. As soon as $D$ approaches 2 the result was always strongly dependent on the selected expansion point.

The aim of this paper is two-fold: First, we reconsider the techniques to perform loop calculations within a "massive scheme", that is on a manifold of finite size and with fixed space dimension $0<D<2$ and $d$. We show that the perturbation series for the effective coupling can be completely summed in $D=2$ and analyze the long-distance properties in this limit. In addition to [33], instead of analytically continuing loop integrals to $D=2$ from below we also perform calculations directly in $2 D$, which need an explicit short-distance (UV)-cutoff. It turns out that results in $D=2$ are independent of the procedure, i.e. they are universal.

Second, we construct a systematic expansion of the effective coupling in powers of $2-D$. Such an expansion is based on our techniques to resum the perturbation series at each order in $2-D$. A first attempt to go beyond $D=2$ has already been made in [33]. However, there, the effect of the boundaries of the finite manifolds was not taken properly into account, a problem that has now been circumvented by considering closed manifolds. We specialize to a toroidal internal topology corresponding to periodic boundary conditions. Of course, the propagator of the perturbation series needs to be modified, and diagrams become more difficult to calculate. Slightly below $D=2$ we expect power-law behavior of the effective coupling. We present a possible ansatz for the exact effective coupling as a function of the internal dimension $D \lesssim 2$, which is consistent with the expansion in $2-D$. However, it remains an open problem to obtain more information about the power-law behavior, to make this expansion unique.

A short account of this work has already appeared in [34].

\section{Model and physical observables}

\subsection{The model}

The problem of a membrane avoiding only a single point (1.4) may at first sight appear artificial. Besides of being a toy-model for the analysis of the more interesting case of full self-avoidance as discussed above, let us point out that it is, too, a special case of another more general problem, which is interesting by its own. Consider a phantom tethered membrane interacting with some $\delta$-potential located at the origin of the configuration space: The Hamiltonian is given by ( $0 \leq$ $D \leq 2)$

$$
\mathcal{H}[\vec{r}]=\frac{1}{2} \int_{x \in \mathcal{M}}(\nabla \vec{r}(x))^{2}+g_{0} \int_{x \in \mathcal{M}} \delta^{d}(\vec{r}(x)),
$$

where any point in the membrane is labeled by some $D$-component vector $x$, and its position in external space is given by the $d$-component field $\vec{r}(x)$,

$$
\vec{r}: x \in \mathbb{R}^{D} \longrightarrow \vec{r}(x) \in \mathbb{R}^{d} .
$$

The partition function is defined as

$$
\mathcal{Z}=\int \mathcal{D}[\vec{r}] \exp (-\mathcal{H}[\vec{r}]) .
$$


To remove the translational 0-mode, we will consider

$$
\mathcal{Z}^{\diamond}=\int \mathcal{D}[\vec{r}] \delta(\vec{r}(y)) \exp (-\mathcal{H}[\vec{r}])
$$

We now discuss (2.1) in more detail: The first term is the elastic energy of the manifold which is entropic in origin. Elasticity and temperature have been scaled to unity. The second term models the interaction of the manifold with a single point at the origin in the $d$-dimensional configurational space. Let us remind [35,33] that the physical interpretation depends on the dimensionality: In the case that $\mathbb{R}^{d}$ is identical to the embedding space, (2.1) describes a phantom crumpled manifold interacting with a single defect as sketched in figure 1. However, setting $d=1$ (2.1) may as well describe a solid-on-solid like fluctuating interface parameterized by some displacement field and interacting with a parallel plane $(D=2)$ as shown in figure 1 .
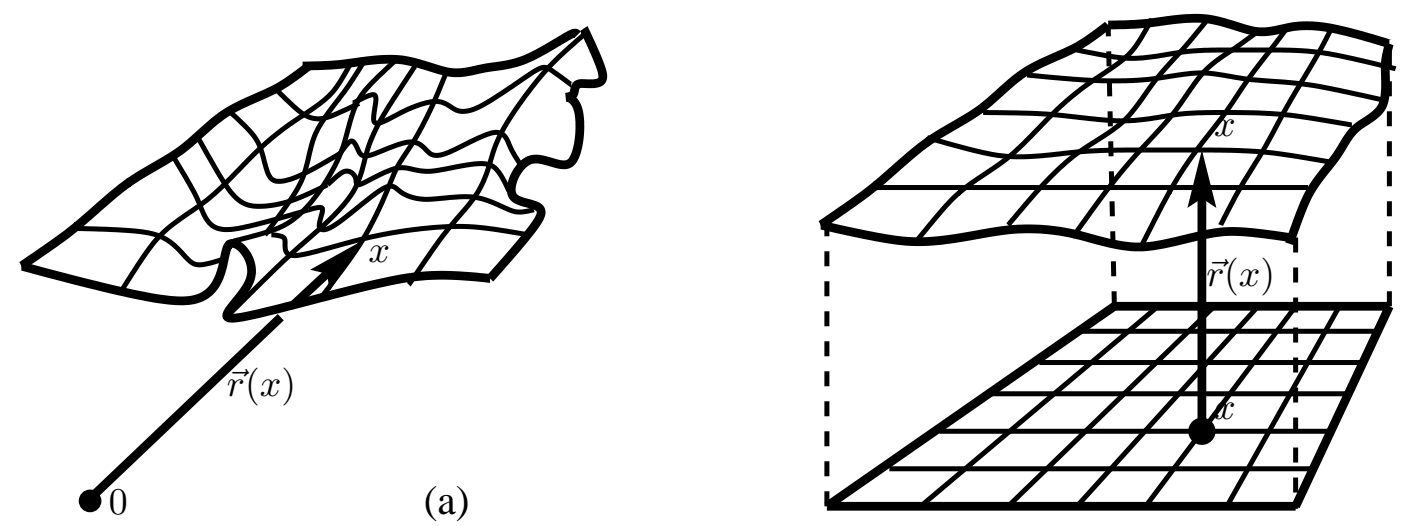

(b)

Figure 1: Left (a): A $D$-dimensional manifold $(D=2)$ interacting with a point in the origin of the configurational space $\mathbb{R}^{d}$. Right (b): A "directed" membrane (interface) interacting with a parallel subspace of same dimension $D$.

The coupling constant $g_{0}$ may either be positive (repulsive interaction) or negative (attractive interaction). We now give the dimensional analysis. In internal space units, the engineering dimensions are

$$
\begin{gathered}
\operatorname{dim}[x]=1 \\
\nu:=\operatorname{dim}[\vec{r}]=\frac{2-D}{2} \\
\varepsilon:=\operatorname{dim}\left[\int_{\mathcal{M}} \mathrm{d}^{D} x \delta^{d}(\vec{r}(x))\right]=D-\nu d .
\end{gathered}
$$

The interaction is naively relevant for $\varepsilon>0$, i.e. $d<d_{c}$ with (see figure 2)

$$
d_{c}=\frac{2 D}{2-D}
$$

irrelevant for $\varepsilon<0$ and marginal for $\varepsilon=0$. It has been shown [35,36] that the model is renormalizable for $0<D<2$ and $\varepsilon \geq 0$. Results for negative $\varepsilon$ are obtained via analytical continuation. One can define the renormalized coupling $g$ as 


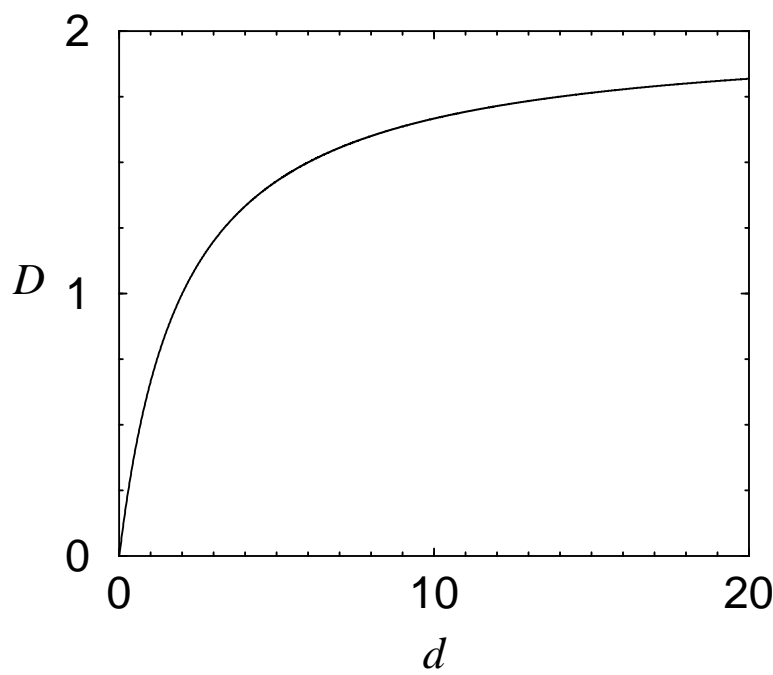

Figure 2: Critical line defined through $\varepsilon=0 \Leftrightarrow d_{c}(D)=\frac{2 D}{2-D}$. The interaction is relevant for points that lie above that line.

$$
g:=\frac{\mathcal{N}}{\mathcal{V}_{\mathcal{M}}}\left[\mathcal{Z}(0)-\mathcal{Z}\left(g_{0}\right)\right] L^{\varepsilon}
$$

where $\mathcal{V}_{\mathcal{M}}$ denotes the internal volume of the manifold. The normalization $\mathcal{N}$ depends on the definition of the path-integral (but not on $L$ ) and is chosen such that

$$
g=g_{0} L^{\varepsilon}+O\left(g_{0}^{2}\right) .
$$

Universal quantities emerge at fixed-points of the $\beta$-function, which is defined as

$$
\beta(g):=-\left.L \frac{\partial g}{\partial L}\right|_{g_{0}} .
$$

The $\beta$-function thus describes, how the effective coupling $g$ changes under scale transformations, while keeping the bare coupling $g_{0}$ fixed. Let us give the 1-loop result, see e.g. [35,36,33].

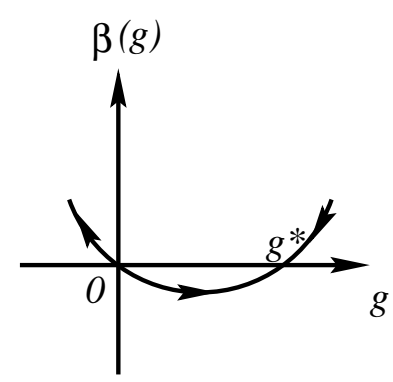

(a)

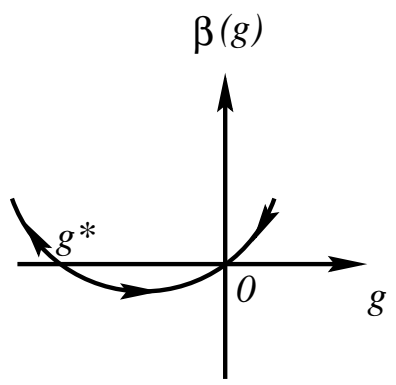

(b)

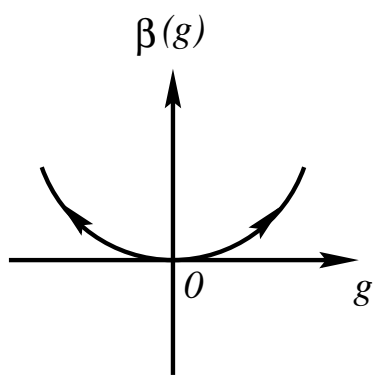

(c)

Figure 3: RG-function and flow for increasing manifold size $L$ for the dimensionless renormalized coupling $g$ : (a) in the case $\varepsilon>0$, (b) in the case $\varepsilon<0$, (c) in the case $\varepsilon=0$. 
It reads

$$
\beta(g)=-\varepsilon g+\frac{1}{2} g^{2}+O\left(g^{3}\right),
$$

where $g$ is the dimensionless renormalized coupling. Apart from the trivial solution, $g=0$, the flow equation given by (2.9) and (2.10) has a non-trivial fixed point at the zero of the $\beta$-function

$$
g^{*}=2 \varepsilon+O\left(\varepsilon^{2}\right)
$$

We shall show below that the scaling behavior is described by the slope of the RG-function at the fixed point, which is universal as a consequence of renormalizabilty. The long-distance behavior is then governed by the $\delta$-interaction as considered in our model (2.1), which is the most relevant operator at large scales. Let us now discuss possible physical situations (see fig. 3):

(a) $\varepsilon>0$ : The RG-flow has an infrared stable fixed point at $g^{*}>0$ and an IR-unstable fixed point at $g=0$. The latter describes an unbinding transition whose critical properties are given by the non-interacting system, while the non-trivial IR stable fixed point determines the long-distance properties of the delocalized state, the long-range repulsive force exerted by the fluctuating manifold on the origin - which we remind may be a point, a line or a plane.

(b) $\varepsilon<0$ : Now, the long-distance behavior is Gaussian, while the unbinding transition occurs at some finite value of the attractive potential, $g^{*}<0$, which corresponds to an infrared unstable fixed point of the $\beta$-function. Below $g^{*}$ the RG-flow is to strong coupling and the manifold is always attracted.

(c) $\varepsilon=0$ : This is the marginal situation, where the transition takes place at $g^{*}=0$; we expect logarithmic corrections to scaling.

These scenarios and possible observables have already been discussed in [33]. Here we want to specialize to the case of a membrane avoiding a single point. It turns out that this situation allows to calculate observables staying non-singular even for $2 D$ membranes and which are accessible to a Monte-Carlo experiment.

\subsection{Plaquettes-density correlation functions}

Interesting physical observables for a membrane avoiding a single point are the plaquettesdensity functions at the repelling point. Generally, these are defined as follows:

$$
\left\langle n^{\ell}\right\rangle_{\diamond}:=\left\langle\prod_{i=1}^{\ell} \int_{x_{i} \in \mathcal{M}} \delta^{d}\left(\vec{r}\left(x_{i}\right)\right)\right\rangle_{\diamond}
$$

where the expectation value $\langle\cdot\rangle_{\diamond}$ is taken within the pinned ensemble as defined in (2.4). The quantity, which is accessible in perturbation theory, is the effective coupling as defined in (2.7). It can be considered as a generating function for observables like (2.12). Let us first show how to 
obtain the constrained partition function (2.4) from (2.7): Since we consider closed manifolds, internal translational invariance implies

$$
\mathcal{Z}^{\diamond} \equiv \mathcal{Z}^{\diamond}\left(g_{0}\right)=-\left.\frac{1}{\mathcal{V}_{\mathcal{M}}} \frac{\partial}{\partial g_{0}}\right|_{L} \mathcal{Z}\left(g_{0}\right)=\left.\frac{1}{\mathcal{N}} \frac{\partial\left(g L^{-\varepsilon}\right)}{\partial g_{0}}\right|_{L},
$$

where $g$ is the renormalized or effective coupling defined in (2.7) and $\mathcal{V}_{\mathcal{M}}$ denotes the internal volume of the membrane. Introducing the dimensionless bare coupling,

$$
z:=g_{0} L^{\varepsilon}
$$

(2.13) can be written in terms of dimensionless quantities as

$$
\mathcal{Z}^{\diamond}\left(g_{0} L^{\varepsilon}\right)=\frac{\partial g}{\partial z}
$$

where $\mathcal{N}$ has been set to unity. In the same way, all observables of the type (2.12) can be easily derived from $g$ according to:

$$
\left\langle n^{\ell}\right\rangle_{\diamond}=\frac{L^{\ell \varepsilon}}{\frac{\partial g}{\partial z}} \frac{\partial^{\ell}}{\partial z^{\ell}}\left(\frac{\partial g}{\partial z}\right)
$$

An observable, which is accessible through Monte-Carlo simulations, should be expressable by universal quantities like the slope of the renormalization $\beta$-function (2.4). All these quantities can be obtained from appropriate derivatives of the effective coupling $g$ with respect to $z$ : The $\beta$-function can be written in terms of the bare coupling as

$$
\beta(z)=-\varepsilon z \frac{\partial g}{\partial z}
$$

(Note that in a slight abuse of notation, we write $\beta(z)=\beta(g(z))$.) The universal slope at the fixed point, which is defined as

$$
\omega:=\left.\frac{\partial \beta(g)}{\partial g}\right|_{g^{*}}
$$

is obtained from

$$
\omega(z)=\frac{-\varepsilon z}{\beta(z)} \frac{\partial \beta(z)}{\partial z}
$$

in the limit $z \rightarrow \infty$. We furthermore need the second derivative of the RG-flow function with respect to the effective coupling, which is defined as

$$
\omega^{\prime}:=\left.\frac{\partial^{2} \beta(g)}{\partial g^{2}}\right|_{g^{*}} \stackrel{z \rightarrow \infty}{=} \frac{-\varepsilon z}{\beta(z)} \frac{\partial \omega(z)}{\partial z} .
$$

Let us now show that the universal slope (2.18) is accessible through the measurement of certain combinations of observables of type (2.12). For this purpose we need the plaquettes-density $(\ell=1)$ and the density-density function $(\ell=2)$, which are obtained after some straight forward, but tedious algebra from the above definitions:

$$
\begin{aligned}
\langle n\rangle_{\diamond} & =\frac{1}{g_{0}}\left(1+\frac{\omega(z)}{\varepsilon}\right) \stackrel{z \rightarrow \infty}{\longrightarrow} \frac{1}{g_{0}}\left(1+\frac{\omega}{\varepsilon}\right) \\
\left\langle n^{2}\right\rangle_{\diamond} & =\frac{1}{g_{0}^{2}}\left(2+\frac{3 \omega(z)}{\varepsilon}+\frac{\omega^{2}(z)}{\varepsilon^{2}}+\frac{\omega^{\prime}(z) \beta(z)}{\varepsilon^{2}}\right) \stackrel{z \rightarrow \infty}{\longrightarrow} \frac{1}{g_{0}^{2}}\left(2+\frac{3 \omega}{\varepsilon}+\frac{\omega^{2}}{\varepsilon^{2}}\right) .
\end{aligned}
$$


These quantities depend on the bare coupling $g_{0}$, which is not accessible. Instead, consider the following ratio:

$$
\frac{\langle n\rangle_{\diamond}}{\sqrt{\left\langle n^{2}\right\rangle_{\diamond}}} \stackrel{z \rightarrow \infty}{=} \sqrt{\frac{1+\frac{\omega}{\varepsilon}}{2+\frac{\omega}{\varepsilon}}},
$$

which obviously is universal.

\subsection{Delocalization transition}

For completeness let us shortly discuss the physical situation at the UV-stable fixed point in figure 3. The fixed point corresponds to a delocalization transition of the manifold, which is at vanishing coupling $g^{*}=0$ for $\varepsilon>0$ and at some finite attractive coupling $g^{*}<0$ for $\varepsilon<0$.

In the localized phase $g<g^{*}$, correlation functions such as $\left\langle[\vec{r}(x)-\vec{r}(y)]^{2}\right\rangle$ and the associated correlation length $\xi_{\|}$(in the $D$-dimensional internal space) should be finite, as well as the radius of gyration $\xi_{\perp}$. Approaching the transition these quantities diverge as [37]

$$
\xi_{\|} \sim\left(g^{*}-g\right)^{-\nu_{\|}} \quad, \quad \xi_{\perp} \sim\left(g^{*}-g\right)^{-\nu_{\perp}} .
$$

Since $\xi_{\perp} \sim \xi_{\|}^{\nu}$, the exponents $\nu_{\|}$and $\nu_{\perp}$ are related through

$$
\nu_{\perp}=\nu_{\|} \nu
$$

$\nu$ being the dimension of the field (2.5).

Furthermore, they are related to the correction-to-scaling exponent $\omega$ :

$$
\nu_{\|}=-\frac{1}{\omega\left(g^{*}\right)}, \quad \nu_{\perp}=-\frac{\nu}{\omega\left(g^{*}\right)} .
$$

Note that $\omega\left(g^{*}\right)<0$ at the transition. Specializing to $(D, d)=(1,1)$, we find

$$
\nu_{\perp}=1 \quad, \quad \nu_{\|}=2 .
$$

These exponents are also valid for the delocalization transition of a 1-dimensional interface from an attractive hard wall in 2-dimensional bulk space [38,37,39,33].

\section{Complete summation of the perturbation series}

\subsection{Perturbation theory}

In (2.2) we saw that physical observables can be derived from the renormalized coupling $g$ (2.7). To obtain $g$ we need the perturbation series of the partition function $\mathcal{Z}$ (2.3):

$$
\mathcal{Z}=\sum_{N=-1}^{\infty} \frac{\left(-g_{0}\right)^{N+1}}{(N+1) !} \mathcal{Z}_{N}
$$

where

$$
\mathcal{Z}_{N}=\left\langle\prod_{i=1}^{N+1} \int_{x_{i}} \tilde{\delta}^{d}\left(r\left(x_{i}\right)\right)\right\rangle_{0}, \quad N \geq 0
$$


and the normalization of the $\delta$-distribution has been chosen to be

$$
\tilde{\delta}^{d}(r(x)):=(4 \pi)^{d / 2} \delta(r(x))=\int_{k} \mathrm{e}^{i k r(x)}
$$

with

$$
\int_{k}:=\pi^{-d / 2} \int \mathrm{d}^{d} k
$$

The advantage of these normalizations is that

$$
\int_{k} \mathrm{e}^{-k^{2}}=1
$$

Accordingly, the perturbation expansion of the effective coupling (2.7) reads

$$
g(z)=\frac{g_{0} L^{\varepsilon}}{\mathcal{V}_{\mathcal{M}}} \sum_{N=0}^{\infty} \frac{\left(-g_{0}\right)^{N}}{(N+1) !}\left\langle\prod_{i=1}^{N+1} \int_{x_{i}} \tilde{\delta}^{d}\left(r\left(x_{i}\right)\right)\right\rangle_{0}
$$

Performing the averages within the Gaussian theory with normalization

$$
\frac{1}{\mathcal{V}_{\mathcal{M}}} \int_{x}\left\langle\tilde{\delta}^{d}(r(x))\right\rangle_{0}=1
$$

one arrives at

$$
g(z)=\frac{g_{0} L^{\varepsilon}}{\mathcal{V}_{\mathcal{M}}} \sum_{N=0}^{\infty} \frac{\left(-g_{0}\right)^{N}}{(N+1) !}\left(\prod_{i=1}^{N+1} \int_{k_{i}} \int_{x_{i}}\right) \tilde{\delta}^{d}\left(\sum_{i=1}^{N+1} k_{i}\right) \mathrm{e}^{\frac{1}{2} \sum_{i, j=1}^{N+1} k_{i} k_{j} C\left(x_{i}-x_{j}\right)}
$$

where

$$
C\left(x_{i}-x_{j}\right):=\frac{1}{2 d}\left\langle\left(r\left(x_{i}\right)-r\left(x_{j}\right)\right)^{2}\right\rangle_{0}
$$

denotes the correlator, and the $\tilde{\delta}^{d}\left(\sum_{i} k_{i}\right)$ stems from the integration over the global translation. Shifting

$$
k_{N+1} \rightarrow k_{N+1}-\sum_{i=1}^{N} k_{i}
$$

the quadratic form in (3.8) transforms to

$$
\begin{aligned}
& \frac{1}{2} \sum_{i, j=1}^{N+1} k_{i} k_{j} C\left(x_{i}-x_{j}\right) \\
& \quad \longrightarrow \sum_{j=1}^{N} k_{N+1} k_{j} C\left(x_{N+1}-x_{j}\right)-\sum_{i, j=1}^{N} k_{i} k_{j} \frac{C\left(x_{N+1}-x_{i}\right)+C\left(x_{N+1}-x_{j}\right)-C\left(x_{i}-x_{j}\right)}{2} .
\end{aligned}
$$

Integrating out the momenta $k_{1}, \ldots, k_{N+1}$ in (3.8), one obtains

$$
g(z)=z \sum_{N=0}^{\infty} \frac{(-z)^{N}}{(N+1) !}\left(\prod_{\ell=1}^{N} \int_{x_{\ell}}\right)(\operatorname{det} \mathfrak{D})^{-d / 2}
$$


where we have factored out $L^{\varepsilon}$ from the loop integration (such that the integrals now run over a torus of size 1), and the matrix elements $\mathfrak{D}_{i j}$ are

$$
\mathfrak{D}_{i j}=\frac{1}{2}\left[C\left(x_{N+1}-x_{i}\right)+C\left(x_{N+1}-x_{j}\right)-C\left(x_{i}-x_{j}\right)\right] .
$$

\subsection{Complete summation in fixed internal space dimension $D=2$}

Let us compute the $N$-loop order of (3.12): The behavior of the propagator $C(x)$ for arguments $x$ large compared to $a$ is of the form

$$
C(x)=c_{0}+\frac{1}{2 \pi} \ln \frac{x}{a},
$$

where $c_{0}$ denotes some positive constant (note $C(x) \geq 0$ ), and the logarithmic growth (for large $x$ ) is universal (see appendix A). In $D=2$ we need an additional short-distance cutoff $a$, which we want to take to 0 . We can (somehow arbitrary) decompose

$$
\operatorname{det} \mathfrak{D}=\left(\prod_{i=1}^{N} \mathfrak{D}_{i i}\right) \operatorname{det} \tilde{\mathfrak{D}} .
$$

In the limit of $a \rightarrow 0$ each $C(x)=\frac{1}{2 \pi} \ln (L / a)+O\left(a^{0}\right)$, such that

$$
\begin{aligned}
& \tilde{\mathfrak{D}}_{i j}=\frac{1}{2}\left[1+\frac{C\left(x_{N+1}-x_{j}\right)-C\left(x_{i}-x_{j}\right)}{C\left(x_{N+1}-x_{i}\right)}\right] \stackrel{a \rightarrow 0}{\longrightarrow} \frac{1}{2}, i \neq j, \\
& \tilde{\mathfrak{D}}_{i i}=1 . \\
&\left(\prod_{\ell=1}^{N} \int_{x_{\ell}}\right)(\operatorname{det} \mathfrak{D})^{-d / 2}=: I_{N}(L / a)=I_{1}^{N}(L / a)\left(\operatorname{det} \tilde{\mathfrak{D}}^{(0)}\right)^{-d / 2} .
\end{aligned}
$$

The matrix $\tilde{\mathfrak{D}}^{(0)}$ denotes the limit $a \rightarrow 0$ of (3.16). It can be written as $\tilde{\mathfrak{D}}^{(0)}=\frac{1}{2}(\mathbb{I}+N \mathbb{P})$, where $\mathbb{I}$ denotes the identity and $\mathbb{P}$ the projector onto $(1,1, \ldots, 1)$, whose image has dimension 1 , such that $\operatorname{det} \tilde{\mathfrak{D}}^{(0)}=\frac{1+N}{2^{N}}$ [33]. Furthermore, to one loop $I_{1}(L / a) \stackrel{a \rightarrow 0}{=} c_{1}\left(\ln \frac{L}{a}\right)^{-d / 2}$, where $c_{1}$ denotes some (finite) constant. One then arrives at

$$
g(z)=z \sum_{N=0}^{\infty} \frac{\left(-z\left(\ln \frac{L}{a}\right)^{-d / 2}\right)^{N}}{N !(1+N)^{d / 2+1}} .
$$

A factor $c_{1} 2^{d / 2}$ has been absorbed into a rescaling of both $z$ and $g$.

\subsection{Asymptotic scaling behavior}

In the following we will analyze the limit of large $z$ (strong repulsion), which also is the scaling behavior of infinitely large membranes. We need an analytical expression for sums like (3.18) in the limit of large $z$. Later, it will turn out that allowing for small deviations $2-D>0$ only slightly more general sums will arise. 
We claim that for all $k, d>0$

$$
\sum_{N=0}^{\infty} \frac{(-z)^{N}}{N !(k+N)^{d / 2}}=\frac{1}{\Gamma\left(\frac{d}{2}\right)} \int_{0}^{\infty} \mathrm{d} r r^{d / 2-1} \exp \left[-z \mathrm{e}^{-r}-k r\right]
$$

This can be proven as follows:

$$
\begin{aligned}
\frac{1}{\Gamma\left(\frac{d}{2}\right)} \int_{0}^{\infty} \mathrm{d} r r^{d / 2-1} \exp \left(-z \mathrm{e}^{-r}-k r\right) & =\frac{1}{\Gamma\left(\frac{d}{2}\right)} \sum_{N=0}^{\infty} \frac{(-z)^{N}}{N !} \int_{0}^{\infty} \mathrm{d} r r^{d / 2-1} \mathrm{e}^{-(N+k) r} \\
& =\frac{1}{\Gamma\left(\frac{d}{2}\right)} \sum_{N=0}^{\infty} \frac{(-z)^{N}}{N !} \frac{\Gamma\left(\frac{d}{2}\right)}{(N+k)^{d / 2}} .
\end{aligned}
$$

This integral-representation is not the most practical for our purpose. It is better to set $r \rightarrow s:=$ $\mathrm{e}^{-r}$ which yields

$$
\sum_{N=0}^{\infty} \frac{(-z)^{N}}{N !(k+N)^{d / 2}}=\frac{1}{\Gamma\left(\frac{d}{2}\right)} \int_{0}^{1} \mathrm{~d} s s^{k-1}(-\ln s)^{d / 2-1} \mathrm{e}^{-s z} .
$$

This formula is already very useful for some purposes. It is still advantageous to make a second variable-transformation $s \rightarrow y:=s z$, yielding

$$
\sum_{N=0}^{\infty} \frac{(-z)^{N}}{N !(k+N)^{d / 2}}=\frac{(\ln z)^{d / 2-1}}{\Gamma\left(\frac{d}{2}\right) z^{k}} \int_{0}^{z} \mathrm{~d} y y^{k-1}\left(1-\frac{\ln y}{\ln z}\right)^{d / 2-1} \mathrm{e}^{-y} .
$$

Finally we remark that we usually have the following combination

$$
f_{k}^{d}(z):=z^{k} \sum_{N=0}^{\infty} \frac{(-z)^{N}}{N !(k+N)^{d / 2}}=\frac{(\ln z)^{d / 2-1}}{\Gamma\left(\frac{d}{2}\right)} \int_{0}^{z} \mathrm{~d} y y^{k-1}\left(1-\frac{\ln y}{\ln z}\right)^{d / 2-1} \mathrm{e}^{-y}
$$

It satisfies the following simple recursion relation, which is helpful to calculate the $\beta$-function:

$$
z \frac{\mathrm{d}}{\mathrm{d} z} f_{k}^{d}(z)=f_{k}^{d-2}(z)
$$

The derivative above can also be rewritten as

$$
z \frac{\mathrm{d}}{\mathrm{d} z} f_{k}^{d}(z)=k f_{k}^{d}(z)-f_{k+1}^{d}(z)
$$

such that one obtains a useful formula in order to isolate the dominant behavior for large $z$ :

$$
f_{k+1}^{d}(z)=k f_{k}^{d}(z)-f_{k}^{d-2}(z) \text {. }
$$

From (3.19) $f_{k}^{d}(z)>0$ for all $k, d>0$ and the behavior for large $z$ is obtained by expanding $\left(1-\frac{\ln y}{\ln z}\right)^{d / 2-1}$ for small $\frac{1}{\ln z}$

$$
\begin{aligned}
f_{k}^{d}(z)=\frac{(\ln z)^{d / 2-1}}{\Gamma\left(\frac{d}{2}\right)}[ & \int_{0}^{\infty} \mathrm{d} y y^{k-1} \mathrm{e}^{-y} \\
& -\frac{1}{\ln z}\left(\frac{d}{2}-1\right) \int_{0}^{\infty} \mathrm{d} y y^{k-1} \ln y \mathrm{e}^{-y} \\
& \left.+O\left(\frac{1}{(\ln z)^{2}}\right)\right]+O\left(\mathrm{e}^{-z}\right)
\end{aligned}
$$


The result is

$$
f_{k}^{d}(z)=\frac{(\ln z)^{d / 2-1} \Gamma(k)}{\Gamma\left(\frac{d}{2}\right)}\left(1-\frac{1}{\ln z} \frac{d-2}{2} \frac{\Gamma^{\prime}(k)}{\Gamma(k)}+\ldots\right) .
$$

With the above notations, the sum (3.18) expressing $g$ as a function of $z$ becomes

$$
g(z, L / a)=\left(\ln \frac{L}{a}\right)^{d / 2} f_{1}^{d+2}\left[z\left(\ln \frac{L}{a}\right)^{-d / 2}\right]
$$

in the limit $D=2$.

It is now easy to analyze the long-distance behavior in this limit. First, we observe that according to (3.27) the effective coupling diverges logarithmically for all external dimensions $d>0$ :

$$
g(z, L / a) \stackrel{z \rightarrow \infty}{\longrightarrow} \frac{\left(\ln \frac{L}{a}\right)^{d / 2}}{\Gamma\left(\frac{d+2}{2}\right)}\left[\ln \left(z\left(\ln \frac{L}{a}\right)^{-d / 2}\right)\right]^{d / 2} .
$$

This is in contrast to the one-loop result as stated in (2.11), which is exact for polymers $(D=1)$ and which stays qualitatively valid as long as $D<2$. This follows from the renormalizability of the theory [35] for sufficiently small $\varepsilon>0$. A finite limit $g(z \rightarrow \infty)=g^{*}$ signals a scale invariant theory. In (3.29) we have found the limiting behavior of the latter. Consequently, we expect the correction-to-scaling exponent $\omega$ to be always zero in $D=2$. In order to check that let us first compute the renormalization $\beta$-function in terms of the bare coupling as in (2.17), which can be immediately derived with the help of relation $(3.23)^{1}$ :

$$
\beta\left(z^{\prime}\right)=-\varepsilon z^{\prime} \frac{\partial g^{\prime}}{\partial z^{\prime}}=-\varepsilon f_{1}^{d}\left(z^{\prime}\right) \stackrel{z^{\prime} \rightarrow \infty}{\longrightarrow} \frac{1}{\Gamma\left(\frac{d}{2}\right)}\left[\ln \left(z^{\prime}\right)\right]^{d / 2-1}
$$

where we have introduced rescaled couplings $z^{\prime}:=g_{0} L^{\varepsilon}\left(\ln \frac{L}{a}\right)^{-d / 2}$ and $g^{\prime}=g\left(\ln \frac{L}{a}\right)^{-d / 2}$. Its derivative with respect to the renormalized coupling is found as a function of the bare coupling (2.19) to be

$$
\omega\left(z^{\prime}\right)=\frac{-\varepsilon z^{\prime}}{\beta\left(z^{\prime}\right)} \frac{\partial \beta\left(z^{\prime}\right)}{\partial z^{\prime}}=-\varepsilon \frac{z^{\prime} \frac{\mathrm{d}}{\mathrm{d} z^{\prime}} f_{1}^{d}\left(z^{\prime}\right)}{f_{1}^{d}\left(z^{\prime}\right)} \stackrel{z^{\prime} \rightarrow \infty}{\longrightarrow} \varepsilon \frac{2-d}{2 \ln \left(z^{\prime}\right)} \stackrel{z^{\prime} \rightarrow \infty}{\longrightarrow} 0 .
$$

Note that the qualitative behavior of the $\beta$-function changes depending on the external dimension $d$, approaching asymptotically zero below $d=2$ and being divergent above.

In the limit of large bare couplings one may as well give the RG-function in terms of the effective (renormalized) coupling simply by inverting the asymptotic expression in (3.29) and inserting it into (3.30), with the result:

$$
\beta(g) \stackrel{z^{\prime} \rightarrow \infty}{\sim}-\varepsilon \frac{\left(\Gamma\left(\frac{d+2}{2}\right)\right)^{1-2 / d}}{\Gamma\left(\frac{d}{2}\right)} g^{1-2 / d} .
$$

\footnotetext{
${ }^{1}$ Note that our definition $\beta\left(z^{\prime}\right)=-\epsilon z^{\prime} \partial g^{\prime} / \partial z^{\prime}$ is strictly speaking equivalent to definining the $\beta$-function as $\beta(g):=\left.\left(-L \frac{\mathrm{d}}{\mathrm{d} L}-a \frac{\mathrm{d}}{\mathrm{d} a}\right)\right|_{g_{0}} g$, instead of (2.9). (Note that the derivative w.r.t. $a$ disappears for $D<2$.) The natural combination in $D=2$ is $z^{\prime}=g_{0} L^{\epsilon}\left(\ln \frac{L}{a}\right)^{-d / 2}$ instead of $z=g_{0} L^{\epsilon}$, and normalizations such that $g^{\prime}\left(z^{\prime}\right)=z^{\prime}+O\left(z^{\prime 2}\right)$ does not explicitly depend on $L$ or $a$. The chosen defintions avoid unnecessary technical complications, but do not change the physics of the problem.
} 


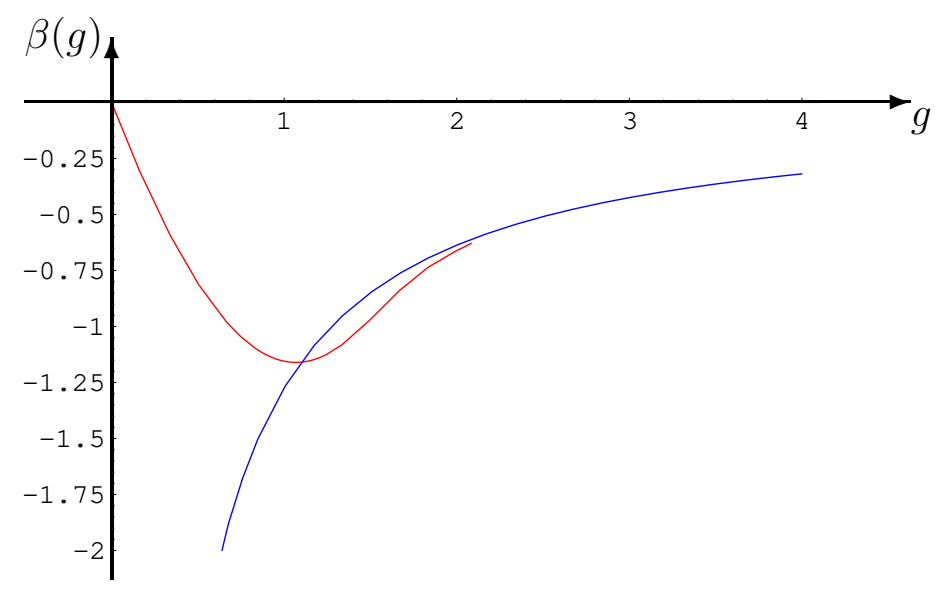

Figure 4: $\beta$-function in terms of the renormalized coupling $g$ truncated at order 160, Pade-resummed, and plotting only that part for which the truncated series converges. (This can e.g. be tested by taking away the last few terms of the series.) This is compared to the asymptotic behavior (3.32) (proportional to $1 / g$ for large $g$ ). $d$ is set to 1 , and we used the diagonal $(80,80)$-Pade approximant, which was find to converge best. (The non-resummed expression starts to diverge already at $g \approx 1.8$ at this order.)

It is interesting to compare the true asymptotic behavior of the completely resummed perturbation series as found above with predictions taking only finite loop orders into account: If one tries to invert (3.18) and truncates it at some finite order, it is at least possible to reach the asymptotic regime (3.32) - however, for large $g$ the truncated $\beta$-function does not converge to the true $\beta$-function and thus strongly deviates from the true behavior. In figure 4 the Pade-resummed truncated $\beta$-function up to order $g^{160}$ in $d=1$ is compared with the asymptotic flow-function. One notices that the truncated $\beta$-function even though improved through a Pade-Resummation hardly gets into touch with the asymptotic regime. The same applies to the slope-function $\omega(g)$, which is not shown in figure 4. Let us finally state the expected behavior of the plaquettes-density functions in the limit of large membranes. For the plaquettes-density at the repelling fixed-point we find in this limit:

$$
\langle n\rangle_{\diamond}=\frac{1}{g_{0}}\left(1+\frac{2-d}{2 \ln z}\right) \stackrel{z \rightarrow \infty}{\sim} \frac{1}{g_{0}} .
$$

Note that in the absence of the repelling interaction this quantity would diverge in this limit. This follows from dimensional grounds, since then

$$
\langle n\rangle_{\diamond} \sim L^{\varepsilon}
$$

In (3.33) we found the largest possible depopulation of monomers at the defect potential in the case of a relevant interaction $(\varepsilon>0)$. As we discussed in (2.2) a measurable quantity should be the following ratio (2.22), which in the case of $2 D$-membranes becomes in the limit $z \rightarrow \infty$ :

$$
\frac{\langle n\rangle_{\diamond}}{\sqrt{\left\langle n^{2}\right\rangle_{\diamond}}} \stackrel{z \rightarrow \infty}{=} \sqrt{\frac{1}{2}}
$$




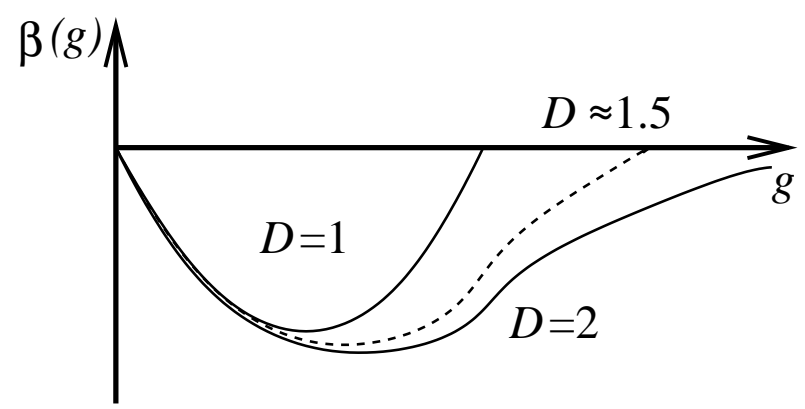

Figure 5: Qualitative behavior for the $\beta$-function in $D=1, D=2$ and result anticipated for $D \approx 1.5$.

which can be compared with the 1-loop prediction (which is exact for polymers):

$$
\frac{\langle n\rangle_{\diamond}}{\sqrt{\left\langle n^{2}\right\rangle_{\diamond}}} \stackrel{z \rightarrow \infty}{=} \sqrt{\frac{2}{3}}, \quad \text { (1-loop) }
$$

\section{Crossover to polymers}

Let us now analyze the theory below $D=2$. Due to the renormalizability in $0<D<2$ and the existence of an $\varepsilon$-expansion we expect the renormalized coupling to reach a finite fixed point in the strong coupling limit as soon as $D<2$. This approach is characterized by a power-law decay of the form

$$
g(z)=g^{*}+S(\ln z) z^{-\omega / \varepsilon}+O\left(z^{-\omega_{1} / \varepsilon}\right),
$$

where $S$ is some scaling-function growing at most sub-exponentially and $\omega_{1}>\omega>0$, with $\omega$ defined in (2.18).

Our ultimate aim is to draw information from an expansion in powers of $2-D$ of the effective coupling about the correction-to-scaling exponent $\omega$ in (4.1) for $D \lesssim 2$. The scale invariant behavior below $D=2$ results in a finite fixed point of the renormalization $\beta$-function as a function of the effective coupling. The qualitative behavior of the $\beta$-function is sketched in fig. (5).

\section{$4.1 \quad(2-D)$-expansion on the torus}

In order to gain information about $g$ below $D=2$ one has to expand the loop integrand $(\operatorname{det} \mathfrak{D})^{-d / 2}(3.12)$ in powers of $2-D$. For convenience, we take $a \rightarrow 0$. The propagator takes in infinite $D$-space the form $C(x)=|x|^{2-D} /\left(S_{D}(2-D)\right)$, where $S_{D}=2 \pi^{D / 2} / \Gamma\left(\frac{D}{2}\right)$ denotes the volume of the $D$-dimensional unit-sphere. The factor $\left(S_{D}(2-D)\right)^{-1}$ replaces $\ln \left(\frac{L}{a}\right)$ and is absorbed into a rescaling of the field and the coupling according to $r \rightarrow r\left(S_{D}(2-D)\right)^{1 / 2}$ and $g_{0} \rightarrow g_{0}\left(S_{D}(2-D)\right)^{d / 2}$, such that the factors of $\left(\ln \frac{L}{a}\right)^{-d / 2}$ in (3.18) and (3.29) disappear. The propagator in the rescaled variable can then be written as

$$
C(x)=1+(2-D) \mathbb{C}(x),
$$

where for convenience of notation we allow $\mathbb{C}(x)$ to depend itself on $D$. 
Of course, on a closed manifold of finite size, $C(x)$ is modified, but the form (4.2) is independent of the shape of the manifold. Accordingly, one may expand the matrix $\mathfrak{D}$ as

$$
\mathfrak{D}=\tilde{\mathfrak{D}}^{(0)}+(2-D) \mathbb{D},
$$

where $\tilde{\mathfrak{D}}^{(0)}$ is defined as before and coincides with the limit $D \rightarrow 2$ when inserting the above $C(x)$ into $\mathfrak{D}$. Moreover, $\mathbb{D}$ is of the same form as $\mathfrak{D}$, but each $C(x)$ has been replaced with $\mathbb{C}(x)$ :

$$
\mathbb{D}_{i j}=\frac{1}{2}\left[\mathbb{C}\left(x_{N+1}-x_{i}\right)+\mathbb{C}\left(x_{N+1}-x_{j}\right)+\mathbb{C}\left(x_{i}-x_{j}\right)\right] .
$$

Then,

$$
\operatorname{det} \mathfrak{D}=\operatorname{det} \tilde{\mathfrak{D}}^{(0)} \exp \left\{\operatorname{Tr}\left[\ln \left(1+(2-D)\left[\tilde{\mathfrak{D}}^{(0)}\right]^{-1} \mathbb{D}\right)\right]\right\},
$$

where $\left[\tilde{\mathfrak{D}}^{(0)}\right]^{-1}=2\left(\mathbb{I}-\frac{N}{N+1} \mathbb{P}\right)$ denotes the inverse matrix of $\tilde{\mathfrak{D}}^{(0)}$.

Denoting

$$
\mathfrak{M}:=\left[\mathfrak{D}^{(0)}\right]^{-1} \mathfrak{D}
$$

we expand the determinant in (4.5) up to fourth order in $2-D$ :

$$
\begin{aligned}
& {[\operatorname{det}(\mathfrak{D})]^{-d / 2}=\left[\operatorname{det}\left(\mathfrak{D}^{(0)}\right)\right]^{-d / 2}\left[1-\frac{d}{2}[(2-D) \operatorname{Tr} \mathfrak{M}\right.} \\
& \left.-\frac{(2-D)^{2}}{2} \operatorname{Tr} \mathfrak{M}^{2}+\frac{(2-D)^{3}}{2} \operatorname{Tr} \mathfrak{M}^{3}-\frac{(2-D)^{4}}{4} \operatorname{Tr} \mathfrak{M}^{4}\right] \\
& +\frac{d^{2}}{8}\left[(2-D)^{2} \operatorname{Tr}^{2} \mathfrak{M}-(2-D)^{3} \operatorname{Tr} \mathfrak{M} \operatorname{Tr} \mathfrak{M}^{2}+(2-D)^{4}\left[\frac{1}{4} \operatorname{Tr}^{2} \mathfrak{M}^{2}+\frac{2}{3} \operatorname{Tr} \mathfrak{M} \operatorname{Tr} \mathfrak{M}^{3}\right]\right] \\
& \left.-\frac{d^{3}}{48}\left[(2-D)^{3} \operatorname{Tr}^{3} \mathfrak{M}-(2-D)^{4} \frac{3}{2} \operatorname{Tr}^{2} \mathfrak{M} \operatorname{Tr} \mathfrak{M}^{2}\right]+\frac{d^{4}}{384}(2-D)^{4} \operatorname{Tr}^{4} \mathfrak{M}\right]+O\left((2-D)^{5}\right) .
\end{aligned}
$$

The first step in the analysis will be to obtain the resummed perturbation series of the effective coupling up to fourth order in $2-D$. That is, we have to insert (4.7) into (4.5), calculate the corresponding loop integrals at each order of perturbation theory, insert the result into (3.12) and sum the appearing series to all orders.

Let us start with the first-order term in $2-D$ from (4.7). We only need $\mathfrak{M}=\left[\mathfrak{D}^{(0)}\right]^{-1} \mathfrak{D}$, which reads

$$
\left(\mathfrak{M}_{i j}\right)=\left(\left[\mathfrak{D}^{(0)}\right]^{-1} \mathfrak{D}\right)_{i j}=\left(2 \mathfrak{D}_{i j}-\frac{2}{1+N} \sum_{k=1}^{N} \mathfrak{D}_{i k}\right) L^{-2 \nu} .
$$

The trace of (4.8) can easily be performed, with the result

$$
\operatorname{Tr} \mathfrak{M}=\left(\frac{2 N}{1+N} \sum_{i=1}^{N} \mathfrak{D}_{i i}-\frac{2}{1+N} \sum_{i=1}^{N} \sum_{k=1}^{N}\left(1-\delta_{i k}\right) \mathfrak{D}_{i k}\right) L^{-2 \nu}
$$




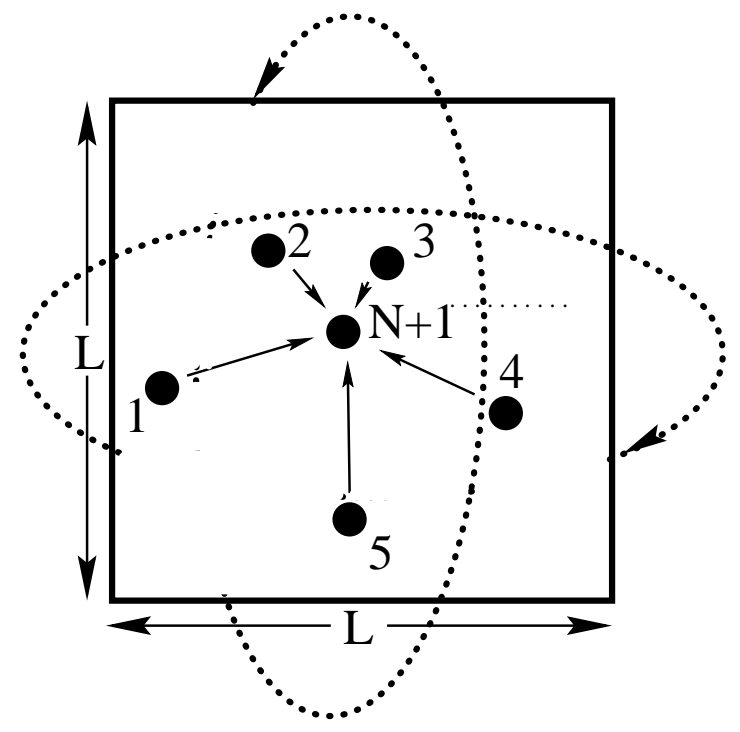

Figure 6: Regularization scheme for the $N$-loop diagrams on manifolds with toroidal topology (periodic boundary conditions). Here: $D=2$

In each order of perturbation theory we have to integrate the expression (4.7) over internal distances. These integrals have to be regularized in the infrared through an appropriate IR cutoff. We are considering a finite manifold of toroidal topology. The precise form of the correlator on the torus will only later enter into the calculation.

To simplify the calculations, we further introduce the following notation:

$$
\overline{f\left(x_{i_{1}}, \ldots, x_{i_{k}}\right)}:=\int_{x_{1}} \ldots \int_{x_{N}} f\left(x_{i_{1}}, \ldots, x_{i_{k}}\right)
$$

with the internal integrations defined as

$$
\int_{x \in \mathcal{M}}=L^{\varepsilon} \int_{x}, \quad \int_{x}:=\text { integral over the torus with } L=1,
$$

such that the overbar in (4.10) can be thought of as an averaging procedure, and especially

$$
\overline{1}=1
$$

Thanks to our regularization prescription the integral of (4.9) over internal points can be replaced by $L^{N D}$ (for the integration measure) times

$$
\begin{aligned}
\overline{\operatorname{Tr} \mathfrak{M}}= & \frac{2 N^{2}}{1+N} \overline{\mathbb{C}\left(x_{N+1}-x_{i}\right)} \\
& -\left(\frac{2 N(N-1)}{1+N}\right)\left(\overline{\mathbb{C}\left(x_{N+1}-x_{i}\right)}-\frac{1}{2} \overline{\mathbb{C}\left(x_{i}-x_{j}\right)}\right) \\
= & \frac{2 N}{1+N} \overline{\mathbb{C}\left(x_{N+1}-x_{i}\right)}+\frac{N(N-1)}{1+N} \overline{\mathbb{C}\left(x_{i}-x_{j}\right)} .
\end{aligned}
$$


Due to the internal symmetry of the closed manifolds which we consider the expression above can be further simplified, since

$$
\overline{\mathbb{C}\left(x_{N+1}-x_{i}\right)}=\overline{\mathbb{C}\left(x_{i}-x_{j}\right)} \equiv \overline{\mathbb{C}(x)} .
$$

Introducing a diagrammatic notation

$$
\text { (. }:=\overline{\mathbb{C}(x)}
$$

the $N$-loop integral reads up to first order in $2-D$

$$
\int(\operatorname{det} D)^{-d / 2}=\mu^{-N \varepsilon}\left(\frac{1+N}{2^{N}}\right)^{-d / 2} \times\left[1-\frac{d}{2}(2-D)\left(N\left(\text {. }_{\text {m }_{\text {mo }}}\right)\right)+O\left((2-D)^{2}\right)\right]
$$

For the further analysis we will not only need (4.13), but also the terms appearing to higher order in $2-D$ in (4.7). We derived expressions like (4.13) for $\operatorname{Tr}^{2} M$ and $\operatorname{Tr} M^{2}$ and all terms up to fourth order in $2-D$ with a Mathematica ${ }^{\mathbb{R}}$-program. It is based on the fact that all terms to appear in the expansion (4.7) are of the form $\operatorname{Tr}^{n} M^{m}$ or products of the latter and therefore can be written as $\mathrm{P}(N) /(N+1)^{k}$, where $n, m, k \in \mathbb{N}$ and $\mathrm{P}(N)$ is some polynomial in $N$. It will turn out soon that it is convenient to expand the polynomial $\mathrm{P}(N)$ in terms of the following base:

$$
\left\{1, N, N(N-1), N(N-1)(N-2), \ldots, \prod_{j=0}^{k}(N-j), \ldots\right\}
$$

We obtain:

$$
\begin{aligned}
\overline{\operatorname{Tr} \mathfrak{M}^{2}}= & \frac{-2 N(N-1)-N(N-1)(N-2)}{1+N} \overline{\mathbb{C}(x)}^{2} \\
& +\frac{2 N+3 N(N-1)+N(N-1)(N-2)}{1+N} \overline{\mathbb{C}^{2}(x)}
\end{aligned}
$$

and

$$
\overline{\operatorname{Tr}^{2} \mathfrak{M}}=\frac{4 N(N-1)+N(N-1)(N-2)}{1+N} \overline{\mathbb{C}(x)}^{2}+\frac{2 N}{1+N} \overline{\mathbb{C}}^{2}(x) .
$$

Diagrammatically, the averages can be rewritten as

$$
\text { (.) }:=\overline{\mathbb{C}}(x)^{2}
$$

and

$$
:=\overline{\mathbb{C}^{2}(x)}
$$

Like in the case of the first order diagram (4.18) and (4.19) are highly simplified as compared to an open manifold, see our treatment in [33]. 
Let us shortly discuss the reason for (4.17): Inserting (4.18) and (4.19) into the perturbation series and summing all loop orders, the following series types will appear:

$$
z \sum_{N=0}^{\infty} \frac{\prod_{i=0}^{k-1}(N-i)(-z)^{N}}{N !(N+1)^{d / 2+j+1}}=(-1)^{k} z^{k} \sum_{N=0}^{\infty} \frac{(-z)^{N}}{N !(N+k)^{d / 2+j+1}} \equiv(-1)^{k} f_{k}^{d+2(j+1)}(z) .
$$

We may therefore identify the resummed series with a function that we know already fairly well, in particular we know its strong coupling behavior. It is furthermore convenient to reduce all functions $f_{k>1}^{d+2(j+1)}(z)$ to sums of functions $f_{1}^{d+2(j+1)}(z)$ exploiting the formula (3.25).

\subsection{Resummed contributions to the expansion in $2-D$ up to fourth order}

We are now almost in the position to state all resummed contributions up to fourth order in $2-D$. Let us first state all necessary diagrams:

$$
=\overline{\mathbb{C}\left(x_{i}-x_{j}\right)}
$$

which contributes to first order in $2-D$. To second order one needs in addition

$$
=\overline{\mathbb{C}^{2}\left(x_{i}-x_{j}\right)} \text {. }
$$

To third order diagrams with new topology are

$$
\begin{aligned}
& \text { smm }=\overline{\mathbb{C}^{3}\left(x_{i}-x_{j}\right)}, \\
& a_{u_{m}}=\overline{\mathbb{C}\left(x_{i}-x_{j}\right) \mathbb{C}\left(x_{j}-x_{k}\right) \mathbb{C}\left(x_{i}-x_{k}\right)} \text {. }
\end{aligned}
$$

Finally, to fourth order arise:

$$
\begin{aligned}
& \text { P }=\overline{\mathbb{C}^{2}\left(x_{i}-x_{j}\right) \mathbb{C}\left(x_{k}-x_{j}\right) \mathbb{C}\left(x_{k}-x_{i}\right)}, \\
& =\overline{\mathbb{C}\left(x_{i}-x_{j}\right) \mathbb{C}\left(x_{k}-x_{j}\right) \mathbb{C}\left(x_{l}-x_{k}\right) \mathbb{C}\left(x_{i}-x_{l}\right)},
\end{aligned}
$$

If one calculates diagrams, it will turn out that it is to some extend more convenient to express the above averages in terms of averages over a connected correlation function, which is defined as

$$
\mathbb{C}_{c}(x):=\mathbb{C}(x)-\overline{\mathbb{C}},
$$

such that for instance

$$
\overline{\mathbb{C}_{c}^{2}}=\overline{\mathbb{C}^{2}}-\overline{\mathbb{C}}^{2}
$$


Furthermore, we will need:

$$
\overline{\mathbb{C}_{c}^{3}}=\overline{\mathbb{C}^{3}}-3 \overline{\mathbb{C}} \overline{\mathbb{C}^{2}}+2 \overline{\mathbb{C}}^{3}
$$

and

$$
\begin{aligned}
\overline{\mathbb{C}_{c}^{\triangle}} & =\overline{\mathbb{C}_{c}\left(x_{i}-x_{j}\right) \mathbb{C}_{c}\left(x_{j}-x_{k}\right) \mathbb{C}_{c}\left(x_{k}-x_{i}\right)} \\
& =\overline{\mathbb{C}\left(x_{i}-x_{j}\right) \mathbb{C}\left(x_{j}-x_{k}\right) \mathbb{C}\left(x_{k}-x_{i}\right)}+3 \overline{\mathbb{C}}^{2} \overline{\mathbb{C}\left(x_{i}-x_{j}\right)}-3 \overline{\mathbb{C}} \overline{\mathbb{C}\left(x_{i}-x_{j}\right) \mathbb{C}\left(x_{j}-x_{k}\right)}-\overline{\mathbb{C}}^{3} \\
& =\overline{\mathbb{C}^{\triangle}}-\overline{\mathbb{C}}^{3}
\end{aligned}
$$

where $x_{i}, x_{j}, x_{k}$ are distinct points, and the average is over their positions. In (4.30) we exploited the symmetry of the closed manifold, and the definition of $\overline{\mathbb{C}^{\triangle}}$ is self-evident. Furthermore, we will need to fourth order in $2-D$ :

$$
\begin{aligned}
& \overline{\mathbb{C}_{c}^{4}}=\overline{\mathbb{C}^{4}}+12 \overline{\mathbb{C}^{2}} \overline{\mathbb{C}}^{2}-4 \overline{\mathbb{C}^{3}} \overline{\mathbb{C}}-3 \overline{\mathbb{C}}^{4}, \\
\overline{\mathbb{C}_{c}^{\diamond}}= & \overline{\mathbb{C}_{c}\left(x_{i}-x_{j}\right) \mathbb{C}_{c}\left(x_{j}-x_{k}\right) \mathbb{C}_{c}\left(x_{k}-x_{l}\right) \mathbb{C}_{c}\left(x_{l}-x_{i}\right)} \\
= & \overline{\mathbb{C}^{\diamond}}+5 \overline{\mathbb{C}}^{4}
\end{aligned}
$$

and

$$
\begin{aligned}
\overline{\mathbb{C}_{c}} & =\overline{\mathbb{C}_{c}^{2}\left(x_{i}-x_{j}\right) \mathbb{C}_{c}\left(x_{i}-x_{k}\right) \mathbb{C}_{c}\left(x_{k}-x_{j}\right)} \\
& =\overline{\mathbb{C}^{\triangleright}}-2 \overline{\mathbb{C}^{\triangle}} \overline{\mathbb{C}}-\overline{\mathbb{C}^{2}} \overline{\mathbb{C}}^{2}+2 \overline{\mathbb{C}}^{4} .
\end{aligned}
$$

Let us now state all terms which appear in the expansion of the renormalized coupling $g(z)$ up to fourth order in $2-D$ according to (4.7). We have to calculate at order $N$ of perturbation theory:

$$
\overline{\operatorname{Tr} \mathfrak{M}}=N \overline{\mathbb{C}} .
$$

Inserting this into the perturbation series and summing up the resulting terms to all orders in $N$ generates the following contributions in the $2-D$-expansion of the renormalized coupling:

$$
\sum_{N=1}^{\infty}\left(\operatorname{det} \mathfrak{D}^{(0)}\right)^{-d / 2} \frac{\overline{\operatorname{Tr} \mathfrak{M}}(-z)^{N+1}}{(N+1) !}=\overline{\mathbb{C}} f_{1}^{d+2}(z)-\overline{\mathbb{C}} f_{1}^{d}(z),
$$

which contributes to first order in $2-D$.

To second order in $2-D$, we have (4.18) providing

$$
\begin{aligned}
\sum_{N=1}^{\infty}\left(\operatorname{det} \mathfrak{D}^{(0)}\right)^{-d / 2} \frac{\overline{\operatorname{Tr} \mathfrak{M}^{2}}(-z)^{N+1}}{(N+1) !}= & 2 \overline{\mathbb{C}_{c}^{2}} f_{1}^{d+4}(z)+\left(-4 \overline{\mathbb{C}_{c}^{2}}+\overline{\mathbb{C}}^{2}\right) f_{1}^{d+2}(z) \\
& +\left(-\overline{\mathbb{C}}^{2}+3 \overline{\mathbb{C}_{c}^{2}}\right) f_{1}^{d}(z)-\overline{\mathbb{C}_{c}^{2}} f_{1}^{d-2}(z)
\end{aligned}
$$

and (4.19) providing

$$
\begin{aligned}
\sum_{N=1}^{\infty}\left(\operatorname{det} \mathfrak{D}^{(0)}\right)^{-d / 2} \frac{\overline{\operatorname{Tr}^{2} \mathfrak{M}}(-z)^{N+1}}{(N+1) !}= & 2 \overline{\mathbb{C}_{c}^{2}} f_{1}^{d+4}(z)-\left(2 \overline{\mathbb{C}_{c}^{2}}+\overline{\mathbb{C}}^{2}\right) f_{1}^{d+2}(z) \\
& +2 \overline{\mathbb{C}}^{2} f_{1}^{d}(z)-\overline{\mathbb{C}}^{2} f_{1}^{d-2}(z)
\end{aligned}
$$


Let us now state the terms at third order in $2-D$, which we derived with the help of a Mathematica $^{\circledR}$ - program ( $N$ is the loop order.):

$$
\begin{aligned}
& \sum_{N=1}^{\infty}\left(\operatorname{det} \mathfrak{D}^{(0)}\right)^{-d / 2} \frac{\overline{\operatorname{Tr} \mathfrak{M}^{3}}(-z)^{N+1}}{(N+1) !}=4\left(\overline{\mathbb{C}_{c}^{3}}-4 \overline{\mathbb{C}_{c}^{\triangle}}\right) f_{1}^{d+4}(z) \\
& +\left(-10 \overline{\mathbb{C}_{c}^{3}}+36 \overline{\mathbb{C}_{c}^{\triangle}}+6 \overline{\mathbb{C}} \overline{\mathbb{C}_{c}^{2}}\right) f_{1}^{d+2}(z) \\
& +\left(9 \overline{\mathbb{C}_{c}^{3}}-32 \overline{\mathbb{C}_{c}^{\triangle}}-12 \overline{\mathbb{C}} \overline{\mathbb{C}_{c}^{2}}+\overline{\mathbb{C}}^{3}\right) f_{1}^{d}(z) \\
& +\left(3 \overline{\mathbb{C}_{c}^{3}}-17 \overline{\mathbb{C}_{c}^{\triangle}}-9 \overline{\mathbb{C}} \overline{\mathbb{C}_{c}^{2}}+\overline{\mathbb{C}}^{3}\right) f_{1}^{d-2}(z) \\
& -3\left(2 \overline{\mathbb{C}_{c}^{\triangle}}+\overline{\mathbb{C}} \overline{\mathbb{C}_{c}^{2}}\right) f_{1}^{d-4}(z)+\overline{\mathbb{C}_{c}^{\triangle}} f_{1}^{d-6}(z), \\
& \sum_{N=1}^{\infty}\left(\operatorname{det} \mathfrak{D}^{(0)}\right)^{-d / 2} \frac{\overline{\operatorname{Tr} \mathfrak{M} \operatorname{Tr} \mathfrak{M}^{2}}(-z)^{N+1}}{(N+1) !}=4\left(\overline{\mathbb{C}_{c}^{3}}-4 \overline{\mathbb{C}_{c}^{\triangle}}\right) f_{1}^{d+6}(z) \\
& +\left(-8 \overline{\mathbb{C}_{c}^{3}}+32 \overline{\mathbb{C}_{c}^{\triangle}}+2 \overline{\mathbb{C}} \overline{\mathbb{C}_{c}^{2}}\right) f_{1}^{d+4}(z) \\
& +\left(6 \overline{\mathbb{C}_{c}^{3}}-20 \overline{\mathbb{C}_{c}^{\triangle}}+2 \overline{\mathbb{C}} \overline{\mathbb{C}_{c}^{2}}-6 \overline{\mathbb{C}}^{3}\right) f_{1}^{d+2}(z) \\
& +\left(-2 \overline{\mathbb{C}_{c}^{3}}+4 \overline{\mathbb{C}_{c}^{\triangle}}-7 \overline{\mathbb{C}} \overline{\mathbb{C}_{c}^{2}}+2 \overline{\mathbb{C}}^{3}\right) f_{1}^{d}(z) \\
& -\left(\overline{\mathbb{C}}^{3}-4 \overline{\mathbb{C}} \overline{\mathbb{C}_{c}^{2}}\right) f_{1}^{d-2}(z)-\overline{\mathbb{C}} \overline{\mathbb{C}_{c}^{2}} f_{1}^{d-4}(z), \\
& \sum_{N=1}^{\infty}\left(\operatorname{det} \mathfrak{D}^{(0)}\right)^{-d / 2} \frac{\overline{\operatorname{Tr}^{3} \mathfrak{M}}(-z)^{N+1}}{(N+1) !}=4\left(\overline{\mathbb{C}_{c}^{3}}-4 \overline{\mathbb{C}_{c}^{\triangle}}\right) f_{1}^{d+6}(z) \\
& +\left(-4 \overline{\mathbb{C}_{c}^{3}}+24 \overline{\mathbb{C}_{c}^{\triangle}}-6 \overline{\mathbb{C}} \overline{\mathbb{C}_{c}^{2}}\right) f_{1}^{d+4}(z) \\
& +\left(-8 \overline{\mathbb{C}_{c}^{\triangle}}+12 \overline{\mathbb{C}} \overline{\mathbb{C}_{c}^{2}}+\overline{\mathbb{C}}^{3}\right) f_{1}^{d+2}(z) \\
& -3\left(\overline{\mathbb{C}}^{3}+2 \overline{\mathbb{C}} \overline{\mathbb{C}_{c}^{2}}\right) f_{1}^{d}(z) \\
& +3 \overline{\mathbb{C}}^{3} f_{1}^{d-2}(z)-\overline{\mathbb{C}}^{3} f_{1}^{d-4}(z) \text {. }
\end{aligned}
$$

To fourth order in $2-D$ we obtain:

$$
\begin{aligned}
& \sum_{N=1}^{\infty}\left(\operatorname{det} \mathfrak{D}^{(0)}\right)^{-d / 2} \frac{\overline{\operatorname{Tr}^{4} \mathfrak{M}}(-z)^{N+1}}{(N+1) !} \\
& =8\left(222 \overline{\mathbb{C}}^{4}+6 \overline{\mathbb{C}}^{2} \overline{\mathbb{C}}_{c}^{2}+3{\overline{\mathbb{C}_{c}^{2}}}^{2}-\overline{\overline{\mathbb{C}}_{c}^{4}}+24 \overline{\mathbb{C}_{c}^{\curvearrowright}}-36 \overline{\mathbb{C}_{c}^{\diamond}}\right) f_{1}^{d+8}(z) \\
& +4\left(804 \overline{\mathbb{C}}^{4}+12 \overline{\mathbb{C}}^{2} \overline{\mathbb{C}_{c}^{2}}+3{\overline{\mathbb{C}_{c}^{2}}}^{2}-2 \overline{\mathbb{C}_{c}^{4}}+72 \overline{\mathbb{C}_{c}^{\triangleright}}-132 \overline{\mathbb{C}_{c}^{\diamond}}-4 \overline{\mathbb{C}} \overline{\mathbb{C}_{c}^{3}}+16 \overline{\mathbb{C}} \overline{\mathbb{C}_{c}^{\triangleright}}\right) f_{1}^{d+6}(z) \\
& -4\left(432 \overline{\mathbb{C}}^{4}-3 \overline{\mathbb{C}}^{2} \overline{\mathbb{C}_{c}^{2}}-6{\overline{\mathbb{C}_{c}^{2}}}^{2}+24 \overline{\mathbb{C}_{c}^{\triangleright}}-72 \overline{\mathbb{C}_{c}^{\diamond}}-8 \overline{\mathbb{C}} \overline{\mathbb{C}_{c}^{3}}+40 \overline{\mathbb{C}} \overline{\mathbb{C}_{c}^{\triangleright}}\right) f_{1}^{d+4}(z) \\
& +\left(287 \overline{\mathbb{C}}^{4}-36 \overline{\mathbb{C}}^{2} \overline{\mathbb{C}_{c}^{2}}-12{\overline{\mathbb{C}_{c}^{2}}}^{2}-48 \overline{\mathbb{C}_{c}^{\diamond}}-16 \overline{\mathbb{C}} \overline{\mathbb{C}_{c}^{3}}+128 \overline{\mathbb{C}} \overline{\mathbb{C}_{c}^{\triangleright}}\right) f_{1}^{d+2}(z) \\
& +\left(4 \overline{\mathbb{C}}^{4}+36 \overline{\mathbb{C}}^{2} \overline{\mathbb{C}_{c}^{2}}-32 \overline{\mathbb{C}} \overline{\mathbb{C}_{c}^{\triangleright}}\right) f_{1}^{d}(z) \\
& +\left(-6 \overline{\mathbb{C}}^{4}-2 \overline{\mathbb{C}}^{2} \overline{\mathbb{C}_{c}^{2}}\right) f_{1}^{d-2}(z)+4 \overline{\mathbb{C}}^{4} f_{1}^{d-4}(z)-\overline{\mathbb{C}}^{4} f_{1}^{d-6}(z)
\end{aligned}
$$




$$
\begin{aligned}
& \sum_{N=1}^{\infty}\left(\operatorname{det} \mathfrak{D}^{(0)}\right)^{-d / 2} \frac{\overline{\operatorname{Tr} \mathfrak{M}^{2} \operatorname{Tr}^{2} \mathfrak{M}}(-z)^{N+1}}{(N+1) !} \\
& =8\left(222 \overline{\mathbb{C}}^{4}+6 \overline{\mathbb{C}}^{2} \overline{\mathbb{C}_{c}^{2}}+3{\overline{\mathbb{C}_{c}^{2}}}^{2}-\overline{\mathbb{C}_{c}^{4}}+24 \overline{\mathbb{C}_{c}^{\triangleright}}-36 \overline{\mathbb{C}_{c}^{\diamond}}\right) f_{1}^{d+8}(z) \\
& +4\left(960 \overline{\mathbb{C}}^{4}+24 \overline{\mathbb{C}}^{2} \overline{\mathbb{C}_{c}^{2}}+{\overline{\mathbb{C}_{c}^{2}}}^{2}-4 \overline{\mathbb{C}_{c}^{4}}+100 \overline{\mathbb{C}_{c}^{\odot}}-156 \overline{\mathbb{C}_{c}^{\diamond}}\right) f_{1}^{d+6}(z) \\
& -4\left(714 \overline{\mathbb{C}}^{4}+20 \overline{\mathbb{C}}^{2} \overline{\mathbb{C}_{c}^{2}}+8{\overline{\mathbb{C}_{c}^{2}}}^{2}-3 \overline{\mathbb{C}_{c}^{4}}+70 \overline{\mathbb{C}_{c}^{\circ}}-116 \overline{\mathbb{C}_{c}^{\diamond}}-4 \overline{\mathbb{C}} \overline{\mathbb{C}_{c}^{3}}+12 \overline{\mathbb{C}} \overline{\mathbb{C}_{c}^{\Delta}}\right) f_{1}^{d+4}(z) \\
& +\left(889 \overline{\mathbb{C}}^{4}+36 \overline{\mathbb{C}}^{2} \overline{\mathbb{C}_{c}^{2}}-2{\overline{\mathbb{C}_{c}^{2}}}^{2}-28 \overline{\mathbb{C}} \overline{\mathbb{C}_{c}^{3}}-4 \overline{\mathbb{C}_{c}^{4}}+80 \overline{\mathbb{C}_{c}^{\triangleright}}-144 \overline{\mathbb{C}_{c}^{\diamond}}+88 \overline{\mathbb{C}} \overline{\mathbb{C}_{c}^{\Delta}}\right) f_{1}^{d+2}(z) \\
& +\left(-99 \overline{\mathbb{C}}^{4}+3 \overline{\mathbb{C}}^{2} \overline{\mathbb{C}}_{c}^{2}+8{\overline{\mathbb{C}_{c}^{2}}}^{2}+16 \overline{\mathbb{C}} \overline{\mathbb{C}_{c}^{3}}-8 \overline{\mathbb{C}_{c}^{\triangleright}}+16 \overline{\mathbb{C}_{c}^{\diamond}}-48 \overline{\mathbb{C}} \overline{\mathbb{C}_{c}^{\Delta}}\right) f_{1}^{d}(z) \\
& +\left(3 \overline{\mathbb{C}}^{4}-11 \overline{\mathbb{C}}^{2} \overline{\mathbb{C}_{c}^{2}}-2{\overline{\mathbb{C}_{c}^{2}}}^{2}-4 \overline{\mathbb{C}} \overline{\mathbb{C}_{c}^{3}}+8 \overline{\mathbb{C}} \overline{\mathbb{C}_{c}^{\triangle}}\right) f_{1}^{d-2}(z) \\
& +\left(-\overline{\mathbb{C}}^{4}-5 \overline{\mathbb{C}}^{2} \overline{\mathbb{C}_{c}^{2}}\right) f_{1}^{d-4}(z)-\overline{\mathbb{C}}^{2} \overline{\mathbb{C}_{c}^{2}} f_{1}^{d-6}(z)
\end{aligned}
$$

$$
\begin{aligned}
& \sum_{N=1}^{\infty}\left(\operatorname{det} \mathfrak{D}^{(0)}\right)^{-d / 2} \frac{\overline{\operatorname{Tr}^{2} \mathfrak{M}^{2}}(-z)^{N+1}}{(N+1) !} \\
& =8\left(222 \overline{\mathbb{C}}^{4}+6 \overline{\mathbb{C}}^{2} \overline{\mathbb{C}_{c}^{2}}+3{\overline{\mathbb{C}_{c}^{2}}}^{2}-\overline{\mathbb{C}_{c}^{4}}+24 \overline{\mathbb{C}_{c}^{\triangleright}}-36 \overline{\mathbb{C}_{c}^{\diamond}}\right) f_{1}^{d+8}(z) \\
& +4\left(1116 \overline{\mathbb{C}}^{4}+36 \overline{\mathbb{C}}^{2} \overline{\mathbb{C}_{c}^{2}}+23{\overline{\mathbb{C}_{c}^{2}}}^{2}-6 \overline{\mathbb{C}_{c}^{4}}+128 \overline{\mathbb{C}_{c}^{\odot}}-180 \overline{\mathbb{C}_{c}^{\diamond}}+4 \overline{\mathbb{C}} \overline{\mathbb{C}_{c}^{3}}-16 \overline{\mathbb{C}} \overline{\mathbb{C}_{c}^{\triangleright}}\right) f_{1}^{d+6}(z) \\
& -4\left(1080 \overline{\mathbb{C}}^{4}+47 \overline{\mathbb{C}}^{2} \overline{\mathbb{C}_{c}^{2}}+28{\overline{\mathbb{C}_{c}^{2}}}^{2}-8 \overline{\mathbb{C}_{c}^{4}}+132 \overline{\overline{\mathbb{C}}_{c}^{\triangleright}}-172 \overline{\overline{\mathbb{C}}_{c}^{\diamond}}+8 \overline{\mathbb{C}} \overline{\mathbb{C}_{c}^{3}}-32 \overline{\mathbb{C}} \overline{\mathbb{C}_{c}^{\Delta}}\right) f_{1}^{d+4}(z) \\
& +\left(2111 \overline{\mathbb{C}}^{4}+148 \overline{\mathbb{C}}^{2} \overline{\mathbb{C}_{c}^{2}}+44{\overline{\mathbb{C}_{c}^{2}}}^{2}+24 \overline{\mathbb{C}} \overline{\mathbb{C}_{c}^{3}}-24 \overline{\mathbb{C}_{c}^{4}}+272 \overline{\mathbb{C}_{c}^{\odot}}-328 \overline{\mathbb{C}_{c}^{\diamond}}-80 \overline{\mathbb{C}} \overline{\mathbb{C}_{c}^{\Delta}}\right) f_{1}^{d+2}(z) \\
& +\left(-538 \overline{\mathbb{C}}^{4}-74 \overline{\mathbb{C}}^{2} \overline{\mathbb{C}}_{c}^{2}+10{\overline{\mathbb{C}_{c}^{2}}}^{2}-8 \overline{\mathbb{C}} \overline{\mathbb{C}_{c}^{3}}+10 \overline{\mathbb{C}_{c}^{4}}-72 \overline{\mathbb{C}_{c}^{\triangleright}}+80 \overline{\mathbb{C}_{c}^{\diamond}}+8 \overline{\mathbb{C}} \overline{\mathbb{C}_{c}^{\Delta}}\right) f_{1}^{d}(z) \\
& +\left(59 \overline{\mathbb{C}}^{4}+20 \overline{\mathbb{C}}^{2}{\overline{\mathbb{C}_{c}^{2}}}_{c}-15{\overline{\mathbb{C}_{c}^{2}}}^{2}-2 \overline{\overline{\mathbb{C}}_{c}^{4}}+8 \overline{\mathbb{C}_{c}^{\triangleright}}-8 \overline{\mathbb{C}_{c}^{\triangleright}}\right) f_{1}^{d-2}(z) \\
& +\left(-2 \overline{\mathbb{C}}^{2} \overline{\mathbb{C}}_{c}^{2}+6{\overline{\mathbb{C}_{c}^{2}}}^{2}\right) f_{1}^{d-4}(z)-{\overline{\mathbb{C}_{c}^{2}}}^{2} f_{1}^{d-6}(z)
\end{aligned}
$$

$$
\begin{aligned}
& \sum_{N=1}^{\infty}\left(\operatorname{det} \mathfrak{D}^{(0)}\right)^{-d / 2} \frac{\overline{\operatorname{Tr} \mathfrak{M} \operatorname{Tr} \mathfrak{M}^{3}}(-z)^{N+1}}{(N+1) !} \\
& =8\left(222 \overline{\mathbb{C}}^{4}+6 \overline{\mathbb{C}}^{2} \overline{\mathbb{C}_{c}^{2}}+3{\overline{\mathbb{C}_{c}^{2}}}^{2}-\overline{\mathbb{C}_{c}^{4}}+24 \overline{\mathbb{C}_{c}^{\triangleright}}-36 \overline{\mathbb{C}_{c}^{\diamond}}\right) f_{1}^{d+8}(z) \\
& +4\left(1038 \overline{\mathbb{C}}^{4}+30 \overline{\mathbb{C}}^{2} \overline{\mathbb{C}_{c}^{2}}+18{\overline{\mathbb{C}_{c}^{2}}}^{2}-5 \overline{\mathbb{C}_{c}^{4}}+114 \overline{\overline{\mathbb{C}}_{c}^{\triangleright}}-168 \overline{\mathbb{C}_{c}^{\diamond}}+2 \overline{\mathbb{C}} \overline{\mathbb{C}_{c}^{3}}-8 \overline{\mathbb{C}} \overline{\mathbb{C}_{c}^{\triangleright}}\right) f_{1}^{d+6}(z) \\
& -2\left(1818 \overline{\mathbb{C}}^{4}+54 \overline{\mathbb{C}}^{2} \overline{\mathbb{C}_{c}^{2}}+39{\overline{\mathbb{C}_{c}^{2}}}^{2}+\overline{\mathbb{C}} \overline{\mathbb{C}_{c}^{3}}-9 \overline{\mathbb{C}_{c}^{4}}+204 \overline{\mathbb{C}_{c}^{\odot}}-294 \overline{\mathbb{C}_{c}^{\diamond}}-22 \overline{\mathbb{C}} \overline{\mathbb{C}_{c}^{\Delta}}\right) f_{1}^{d+4}(z) \\
& +\left(1583 \overline{\mathbb{C}}^{4}+48 \overline{\mathbb{C}}^{2} \overline{\mathbb{C}_{c}^{2}}+36{\overline{\mathbb{C}_{c}^{2}}}^{2}-\overline{\mathbb{C}} \overline{\mathbb{C}_{c}^{3}}-6 \overline{\mathbb{C}_{c}^{4}}+186 \overline{\mathbb{C}_{c}^{\odot}}-258 \overline{\mathbb{C}_{c}^{\diamond}}+8 \overline{\mathbb{C}} \overline{\mathbb{C}_{c}^{\Delta}}\right) f_{1}^{d+2}(z) \\
& +\left(-358 \overline{\mathbb{C}}^{4}-21 \overline{\mathbb{C}}^{2} \overline{\mathbb{C}_{c}^{2}}-6{\overline{\mathbb{C}_{c}^{2}}}^{2}+6 \overline{\mathbb{C}} \overline{\mathbb{C}_{c}^{3}}-48 \overline{\mathbb{C}_{c}^{\infty}}+60 \overline{\mathbb{C}_{c}^{\triangleright}}-37 \overline{\mathbb{C}} \overline{\mathbb{C}_{c}^{\triangleright}}\right) f_{1}^{d}(z) \\
& +\left(35 \overline{\mathbb{C}}^{4}+12 \overline{\mathbb{C}}^{2} \overline{\mathbb{C}_{c}^{2}}-3 \overline{\mathbb{C}} \overline{\mathbb{C}_{c}^{3}}+6 \overline{\mathbb{C}_{c}^{\triangleright}}-6 \overline{\mathbb{C}_{c}^{\diamond}}+23 \overline{\mathbb{C}} \overline{\mathbb{C}_{c}^{\triangleright}}\right) f_{1}^{d-2}(z) \\
& +\left(-3 \overline{\mathbb{C}}^{2}-7 \overline{\mathbb{C}} \overline{\mathbb{C}_{c}^{\triangle}}\right) f_{1}^{d-4}(z)+\overline{\mathbb{C}} \overline{\mathbb{C}_{c}^{\triangleright}} f_{1}^{d-6}(z)
\end{aligned}
$$




$$
\begin{aligned}
& \sum_{N=1}^{\infty}\left(\operatorname{det} \mathfrak{D}^{(0)}\right)^{-d / 2} \frac{\overline{\operatorname{Tr} \mathfrak{M}^{4}}(-z)^{N+1}}{(N+1) !} \\
& =8\left(222 \overline{\mathbb{C}}^{4}+6 \overline{\mathbb{C}}^{2} \overline{\mathbb{C}}_{c}^{2}+3{\overline{\mathbb{C}_{c}^{2}}}^{2}-\overline{\overline{\mathbb{C}}_{c}^{4}}+24 \overline{\mathbb{C}_{c}^{\triangleright}}-36 \overline{\mathbb{C}_{c}^{\diamond}}\right) f_{1}^{d+8}(z) \\
& +4\left(1116 \overline{\mathbb{C}}^{4}+36 \overline{\mathbb{C}}^{2} \overline{\mathbb{C}_{c}^{2}}+23{\overline{\mathbb{C}_{c}^{2}}}^{2}-6 \overline{\mathbb{C}_{c}^{4}}+128 \overline{\mathbb{C}_{c}^{\curvearrowright}}-180 \overline{\overline{\mathbb{C}}_{c}^{\diamond}}+4 \overline{\mathbb{C}^{3}} \overline{\mathbb{C}_{c}^{3}}-16 \overline{\mathbb{C}} \overline{\mathbb{C}_{c}^{\triangle}}\right) f_{1}^{d+6}(z) \\
& -4\left(1110 \overline{\mathbb{C}}^{4}+39 \overline{\mathbb{C}}^{2} \overline{\mathbb{C}_{c}^{2}}+31{\overline{\mathbb{C}_{c}^{2}}}^{2}+10 \overline{\mathbb{C}} \overline{\mathbb{C}_{c}^{3}}-7 \overline{\mathbb{C}_{c}^{4}}+136 \overline{\mathbb{C}_{c}^{\triangleright}}-178 \overline{\mathbb{C}_{c}^{\diamond}}-36 \overline{\mathbb{C}} \overline{\mathbb{C}_{c}^{\triangle}}\right) f_{1}^{d+4}(z) \\
& +\left(2473 \overline{\mathbb{C}}^{4}+72 \overline{\mathbb{C}}^{2} \overline{\mathbb{C}_{c}^{2}}+82{\overline{\mathbb{C}_{c}^{2}}}^{2}+36 \overline{\mathbb{C}} \overline{\mathbb{C}_{c}^{3}}-16 \overline{\mathbb{C}_{c}^{4}}+304 \overline{\mathbb{C}_{c}^{\triangleright}}-396 \overline{\mathbb{C}_{c}^{\diamond}}-128 \overline{\mathbb{C}} \overline{\mathbb{C}_{c}^{\triangle}}\right) f_{1}^{d+2}(z) \\
& +\left(-955 \overline{\mathbb{C}}^{4}-12 \overline{\mathbb{C}}^{2} \overline{\mathbb{C}_{c}^{2}}-36{\overline{\mathbb{C}_{c}^{2}}}^{2}-12 \overline{\mathbb{C}} \overline{\mathbb{C}_{c}^{3}}+5 \overline{\mathbb{C}_{c}^{4}}-92 \overline{\mathbb{C}_{c}^{\triangleright}}+154 \overline{\mathbb{C}_{c}^{\diamond}}+68 \overline{\mathbb{C}} \overline{\mathbb{C}_{c}^{\triangleright}}\right) f_{1}^{d}(z) \\
& +\left(288 \overline{\mathbb{C}}^{4}+12{\overline{\mathbb{C}_{c}^{2}}}^{2}-\overline{\mathbb{C}_{c}^{4}}+12 \overline{\mathbb{C}_{c}^{\curvearrowright}}-47 \overline{\mathbb{C}_{c}^{\diamond}}-24 \overline{\mathbb{C}} \overline{\mathbb{C}_{c}^{\triangleright}}\right) f_{1}^{d-2}(z) \\
& +\left(-60 \overline{\mathbb{C}}^{4}-2{\overline{\mathbb{C}_{c}^{2}}}^{2}+10 \overline{\mathbb{C}_{c}^{\diamond}}+4 \overline{\mathbb{C}} \overline{\mathbb{C}_{c}^{\triangleright}}\right) f_{1}^{d-4}(z)+\left(6 \overline{\mathbb{C}}^{4}-\overline{\mathbb{C}_{c}^{\diamond}}\right) f_{1}^{d-6}(z) \text {. }
\end{aligned}
$$

\subsection{Renormalized coupling}

Combining (3.12), (4.7) and the results (4.35)-(4.45) from the preceeding subsection we may now give the exact renormalized coupling to fourth order in $2-D$. For the sake of compactness, we introduce a new notation: Since all series contributions are of the form as stated in (4.22), we introduce vectors $\mathbb{M}$ such that

$$
\begin{aligned}
& \sum_{N=1}^{\infty}\left(\operatorname{det} \mathfrak{D}^{(0)}\right)^{-d / 2} \frac{\overline{\prod_{i=1}^{l}\left(\operatorname{Tr} \mathfrak{M}^{n_{i}}\right)^{m_{i}}}(-z)^{N}}{(N+1) !} \equiv \sum_{j=\min }^{\max } \mathbb{M}_{\left(\begin{array}{c}
m_{1} m_{2} \cdots m_{l} \\
n_{1} n_{2} \cdots n_{l}
\end{array}\right)} f_{1}^{d+2 j}(z) \\
& \equiv \mathbb{M}_{\left(\begin{array}{llll}
m_{1} & m_{2} & \cdots & m_{l} \max _{2} \\
n_{1} & n_{2} & \cdots & n_{l} \mid \min
\end{array}\right)} f_{1}^{d+2 j}(z),
\end{aligned}
$$

where max and min are some integers, and summation over the index $j$ is implicit. Inserting the results for the resummed series contributions into (4.7) we find for the renormalized coupling to fourth order in $2-D$ :

$$
\begin{aligned}
& g(z)=f_{1}^{d+2}(z)-(2-D) \frac{d}{2} \mathbb{M}_{\left(\begin{array}{l}
1 \\
1
\end{array} \mid \begin{array}{l}
1 \\
0
\end{array}\right)} f_{1}^{d+2 j}(z)
\end{aligned}
$$

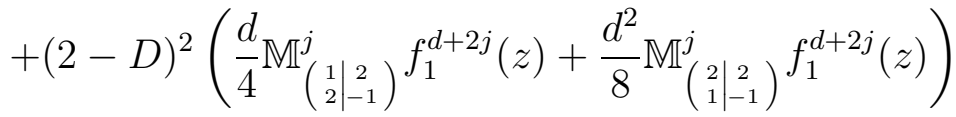

$$
\begin{aligned}
& -(2-D)^{3}\left(\frac{d}{4} \mathbb{M}_{\left(\begin{array}{lll}
1 & 2 \\
3 & -3
\end{array}\right)}^{j} f_{1}^{d+2 j}(z)+\frac{d^{2}}{8} \mathbb{M}_{\left(\begin{array}{ll|l}
1 & 1 & 3 \\
1 & 2 & -2
\end{array}\right)}^{j} f_{1}^{d+2 j}(z)+\frac{d^{3}}{48} \mathbb{M}_{\left(\begin{array}{lll}
3 & 3 \\
1 & S_{-2}
\end{array}\right)}^{j} f_{1}^{d+2 j}(z)\right) \\
& +(2-D)^{4}\left(\frac{d}{8} \mathbb{M}_{\left(\begin{array}{l|l}
4 & 4 \\
1 & -3
\end{array}\right)}^{j} f_{1}^{d+2 j}(z)+\frac{d^{2}}{8}\left(\frac{1}{4} \mathbb{M}_{\left(\begin{array}{l}
2 \\
2
\end{array} A_{-3}\right.}^{j} f_{1}^{d+2 j}(z)+\frac{2}{3} \mathbb{M}_{\left(\begin{array}{ll|l}
1 & 1 & 4 \\
1 & 3 & -3
\end{array}\right)}^{j} f_{1}^{d+2 j}(z)\right)\right. \\
& \left.+\frac{d^{3}}{32} \mathbb{M}_{\left(\begin{array}{ll|l}
2 & 1 & 4 \\
1 & 2 & -3
\end{array}\right)}^{j} f_{1}^{d+2 j}(z)+\frac{d^{4}}{384} \mathbb{M}_{\left(\begin{array}{l|l}
4 & 4 \\
1 & -3
\end{array}\right)}^{j} f_{1}^{d+2 j}(z)\right)+O(2-D)^{5} .
\end{aligned}
$$

The vector entries $\mathbb{M}^{j}$ are to be be taken from subsection 4.2.

It is more convenient to discuss instead of $g(z)$ an integral transform. From the expansion of $f_{k}^{d}(z)$, namely

$$
f_{1}^{d+2 j}(z)=\frac{z}{\Gamma\left(\frac{d}{2}\right)} \int_{0}^{\infty} \mathrm{d} r r^{d / 2+j-1} \exp \left[-z \mathrm{e}^{-r}-r\right]
$$


and the structure of the expansion of $g(z)$ in powers of $2-D$ and the integral representation of the $f_{1}^{d+2 j}$ it follows that the exact renormalized coupling can be written as

$$
g(z) \equiv g(D, z)=z \int_{0}^{\infty} \mathrm{d} r \tilde{g}(r) \exp \left[-z \mathrm{e}^{-r}-r\right]
$$

where $\tilde{g}(r)$ is of the form

$$
\tilde{g}(r)=r^{d / 2}\left[\frac{1}{\Gamma\left(\frac{d+2}{2}\right)}+(2-D) \sum_{n=0}^{\infty} \sum_{j=-n_{\max }}^{n} p_{n_{j}} r^{j}(2-D)^{n}\right] .
$$

\subsection{Guessing the exact $\tilde{g}(r)$}

Let us try to gain more information about the power-law behavior in (4.1), that is about the expansion in $2-D$ of the correction-to-scaling exponent $\omega$. Power-law behavior forces the series (4.50) to turn into some exponentially decaying function $\tilde{g}(r)$ as can be seen from the asymptotic form of $g(z)$ :

$$
g(z) \simeq \mathcal{A}+\mathcal{B} z^{-\omega / \varepsilon}=z \int_{0}^{\infty} \mathrm{d} r \mathrm{e}^{-z \mathrm{e}^{-r}-r}\left(\mathcal{A}+\frac{\mathcal{B} \mathrm{e}^{-r \omega / \epsilon}}{\Gamma\left(1+\frac{\omega}{\varepsilon}\right)}\right)+O\left(\mathrm{e}^{-z}\right) .
$$

In order to check the latter equation note that

$$
\begin{aligned}
f_{1+\frac{\omega}{\varepsilon}}^{2}(z) & =z^{1+\frac{\omega}{\varepsilon}} \int_{0}^{\infty} \mathrm{d} r \exp \left[-z \mathrm{e}^{-r}-(1+\omega / \varepsilon) r\right]=\Gamma\left(1+\frac{\omega}{\varepsilon}\right)+O\left(\mathrm{e}^{-z}\right) \\
\Rightarrow z^{-\omega / \varepsilon} & =\frac{z}{\Gamma\left(1+\frac{\omega}{\varepsilon}\right)} \int_{0}^{\infty} \mathrm{d} r \exp \left[-z \mathrm{e}^{-r}-(1+\omega / \varepsilon) r\right]+O\left(\mathrm{e}^{-z}\right) \\
& =\frac{z}{\Gamma\left(1+\frac{\omega}{\varepsilon}\right)} \sum_{n=0}^{\infty} \int_{0}^{\infty} \mathrm{d} r \frac{(-\omega / \varepsilon r)^{n}}{n !} \exp \left[-z \mathrm{e}^{-r}-r\right]+O\left(\mathrm{e}^{-z}\right) \\
& =\frac{1}{\Gamma\left(1+\frac{\omega}{\varepsilon}\right)} \sum_{n=0}^{\infty} \frac{(-\omega / \varepsilon)^{n}}{n !} f_{1}^{2(n+1)}(z)+O\left(\mathrm{e}^{-z}\right)
\end{aligned}
$$

where it is understood that $\omega$ is expanded in powers of $2-D$.

Let us now test a possible form of the exact $\tilde{g}(r)$. It should satisfy the following properties:

(i) In the limit of $D=2$ the exact result $r^{d / 2} / \Gamma\left(\frac{d+2}{2}\right)$ emerges.

(ii) For $D<2$ the corresponding $g(z)$ has a finite fixed-point value together with a strong coupling expansion. Especially, the ansatz should interpolate to the limit $D=1$, which corresponds to a Gaussian polymer closed to form a ring. The strong coupling expansion of the renormalized coupling of a closed chain interacting with a $\delta$-potential is easily obtained from the factorizability of loop integrals in $D=1$ (see for instance [33]). The result is:

$$
g(z)=\varepsilon\left[1+\sum_{n=1}^{\infty}\left(-\frac{1}{\Gamma(\varepsilon) z}\right)^{n} \frac{1}{\Gamma(1-n \varepsilon)}\right]
$$


(iii) It is consistent with the expansion (4.47).

The (non-unique) ansatz is

$$
\tilde{g}(r)=\mathcal{C}\left(\frac{1-\mathcal{S}(D, r) \mathrm{e}^{-\frac{\omega}{\varepsilon} r}}{\omega / \varepsilon}\right)^{d / 2}
$$

where $\mathcal{S}(D, r)$ is analytic in $D=2$ of the form

$$
\mathcal{S}(D, r)=1+\frac{\omega}{\epsilon} r \sum_{n=1}^{\infty} \mathcal{S}_{n}(r)(2-D)^{n}
$$

and each $\mathcal{S}_{n}(r)$ has a Laurent expansion

$$
\mathcal{S}_{n}(r)=\sum_{j=-n_{\min }}^{n_{\max }} s_{n, j} r^{j}
$$

Note, that in the limit of $D \rightarrow 2$, the expression (4.54) gives $\mathcal{C} r^{d / 2}$, while for $D<2$ it yields upon integration the form (4.1), ensuring both properties (i) and (ii). Let us finally check consistency with the expansion (4.47) up to the second order in $2-D$ : Inserting

$$
\omega / \varepsilon=\omega_{2}(2-D)^{2}+O(2-D)^{3}
$$

(the linear term in $(2-D)$ has to vanish $\left.{ }^{2}\right)$ into the ansatz (4.54) and expanding to second order in $2-D$ provides

$$
\tilde{g}(r)=\mathcal{C} r^{d / 2}\left[1-\frac{d}{2}\left(\mathcal{S}_{1}(r)(2-D)+\left(\frac{\omega_{2}}{2} r-\frac{d-2}{4} \mathcal{S}_{1}(r)^{2}+\mathcal{S}_{2}(r)\right)(2-D)^{2}+\cdots\right)\right] .
$$

Explicitly, (4.47) becomes to second order in $2-D$

$$
\begin{aligned}
g(z)= & f_{1}^{d+2}(z)-(2-D) \frac{d}{2}\left[\overline{\mathbb{C}} f_{1}^{d+2}(z)-\overline{\mathbb{C}} f_{1}^{d}(z)\right] \\
& +(2-D)^{2} \frac{d}{4}\left[2 \overline{\mathbb{C}_{c}^{2}} f_{1}^{d+4}(z)+\left(\overline{\mathbb{C}}^{2}-4 \overline{\mathbb{C}_{c}^{2}}\right) f_{1}^{d+2}(z)+\left(3 \overline{\mathbb{C}_{c}^{2}}-\overline{\mathbb{C}}^{2}\right) f_{1}^{d}(z)-\overline{\mathbb{C}_{c}^{2}} f_{1}^{d-2}(z)\right] \\
& +(2-D)^{2} \frac{d^{2}}{8}\left[2 \overline{\mathbb{C}_{c}^{2}} f_{1}^{d+4}(z)-\left(2 \overline{\mathbb{C}_{c}^{2}}+\overline{\mathbb{C}}^{2}\right) f_{1}^{d+2}(z)+2 \overline{\mathbb{C}}^{2} f_{1}^{d}(z)-\overline{\mathbb{C}}^{2} f_{1}^{d-2}(z)\right] \\
& +O(2-D)^{3} .
\end{aligned}
$$

From this, the first coefficients of the $(2-D)$-expansion of $\tilde{g}(r)$ are obtained. They read

$$
\begin{aligned}
\tilde{g}(r)=\frac{r^{d / 2}}{\Gamma\left(\frac{d+2}{2}\right)}\{1 & +(2-D) \frac{d}{2} \overline{\mathbb{C}}\left(1-\frac{d}{2 r}\right)-(2-D)^{2}\left[\frac{d}{2} \overline{\mathbb{C}_{c}^{2}} r+\frac{d}{4}\left(\overline{\mathbb{C}}^{2}-4 \overline{\mathbb{C}_{c}^{2}}\right)-\frac{d^{2}}{8}\left(2 \overline{\mathbb{C}_{c}^{2}}+\overline{\mathbb{C}}^{2}\right)\right. \\
& \left.\left.+\left(\frac{d^{2}}{8}\left(-\overline{\mathbb{C}}^{2}+3 \overline{\mathbb{C}_{c}^{2}}\right)+\frac{d^{3}}{8} \overline{\mathbb{C}}^{2}\right) r^{-1}-\frac{d^{2}}{8}\left(\frac{d}{2}-1\right)\left(\overline{\mathbb{C}_{c}^{2}}+\frac{d}{2} \overline{\mathbb{C}}^{2}\right) r^{-2}\right]\right\} .(4.60)
\end{aligned}
$$

\footnotetext{
${ }^{2}$ This is due to the fact that the order $(2-D)$ term in $g(z)$ scales identically in $z$ as the leading term. Only the order $(2-D)^{2}$ diverges more strongly.
} 
Comparing (4.58) and (4.60), one identifies $\mathcal{C}=1 / \Gamma\left(\frac{d+2}{2}\right), \mathcal{S}_{1}=-\overline{\mathbb{C}}\left(1-\frac{d}{2} \frac{1}{r}\right)$ and $\omega_{2}=2 \overline{\mathbb{C}_{c}^{2}}$, where $\mathbb{C}_{c}(x):=\mathbb{C}(x)-\overline{\mathbb{C}}$. Note that the terms proportional to $\overline{\mathbb{C}}^{2}$ in $\mathcal{S}_{2}(r)$ mostly cancel with $\mathcal{S}_{1}(r)^{2}$, a sign that the ansatz catches some structure.

The diagrams to be calculated at this order are $\overline{\mathbb{C}}$ and $\overline{\mathbb{C}_{c}^{2}}$ (see appendix B). On a manifold of toroidal shape, which is equivalent to periodic boundary conditions, two discrete sums have to be evaluated:

$$
\begin{aligned}
& \overline{\mathbb{C}}=\frac{S_{D}}{4 \pi^{2}}\left[\sum_{k \in \mathbb{Z}^{D}, k \neq 0} \frac{1}{\vec{k}^{2}}-\frac{2 \pi}{(2-D)}\right]=-0.44956+0.3583(2-D)+O(2-D)^{2} \\
& \overline{\mathbb{C}_{c}^{2}}=\frac{S_{D}^{2}}{16 \pi^{4}} \sum_{k \in \mathbb{Z}^{D}, k \neq 0} \frac{1}{\vec{k}^{4}}=0.152661+O(2-D) .
\end{aligned}
$$

With the results given above, this leads to

$$
\omega=2 \epsilon \overline{\mathbb{C}_{c}^{2}}(2-D)^{2}+O(2-D)^{3}=0.305322 \epsilon(2-D)^{2}+O(2-D)^{3},
$$

which can be compared to the exact result for $D=1$ (polymers): $\omega=\epsilon$. As a caveat, note that the above scheme is not unambiguous in the sense that the second order term proportional to $r$ in (4.61) could in principle either be attributed to $\omega_{2}$ or $\mathcal{S}_{2}$. However, any ansatz in (4.54) will provide an $\omega$, whose expansion starts at least quadratically in $2-D$. Though (4.54) is the best ansatz that could yet be found ensuring properties (i)-(iii), the precise form of constraints on the scaling function $\mathcal{S}$ remains to be discussed in order to settle this question.

\section{Conclusion}

In this work we refined the analysis of a $D$-dimensional elastic manifold interacting by some $\delta$-potential with a fixed point in embedding space. Starting from the perturbation expansion of the effective coupling of the problem, in a first step, we performed a new calculation using a modified regularization prescription: Evaluating loop integrals in fixed space dimension on a manifold of finite size enforced the introduction of a microscopic cutoff as soon as $D=2$. This way, we recovered the complete summability of the perturbation theory in this limit and confirmed the strong coupling behavior as found previously in an analytic continuation from below $D=2$. In the strong coupling limit, corresponding to strong repulsion or equivalently to large membrane sizes, the effective coupling diverges logarithmically as a function of the bare coupling $z$ yielding a vanishing correction-to-scaling exponent $\omega$. Analyzing the $\mathrm{RG} \beta$-function we found that it tends to zero at infinite bare coupling $z$ as $0 \leq d<2$. The renormalization group flow then tends to a fixed point, and the theory becomes scale invariant in this limit. Due to the logarithmic divergence of the effective coupling, however, the corresponding zero of the $\beta$-function in terms of the latter is, too, shifted to infinity. This is a quite remarkable result showing that the scaling behavior of the system is accessible only to an all order treatment and deviates qualitatively from any finite loop expansion, be it within a minimal subtraction scheme or at finite $\varepsilon$. Especially, the logarithmic growth of the effective coupling signals the limiting behavior of a scale-invariant theory.

The result in $D=2$ is completely independent of the regularization procedure. This does no 
longer hold true beyond the leading order, which should be accessible to an expansion in $2-D$. We constructed its first order in a specific regularization scheme in [33]. While this reproduces qualitatively correctly the known result in $D=1$, it suffers from a renormalization scheme, which neglects the boundaries of a finite manifold. We used a hard cutoff in position space, while working with the infinite $D$-space correlator. It seems that only in an $\varepsilon$-expansion this procedure is systematic.

Now, in a second step of we have overcome this problem by constructing the $2-D$-expansion on a manifold of toroidal shape of finite size, thus imposing periodic boundary conditions on the field. There is no further infrared cutoff necessary. We have carried out the expansion of the renormalized coupling up to fourth order in $2-D$, revealing the general structure of the expansion. It is important to point out that in considering $g$ as a function of the bare coupling, the limits $D \rightarrow 2$ and strong coupling $(z \rightarrow \infty)$ can not be interchanged. While $g$ tends to infinity as $z$ does in $D=2$, we expect finiteness of this limit as soon as $2-D>0$ and the existence of a strong coupling expansion as found for polymers $(D=1)$. We were able to guess an exact $g(D, z)$ as a function of $z$ and the internal dimension $D$, which satisfies these properties and which can be reconciled with the available expansion in $2-D$ by an appropriate matching of its free parameters. Though it turned out that due to an ambiguity in the matching of parameters the precise power-law behavior of the effective coupling below $D=2$ can not yet be isolated, we found that for closed manifolds the expansion of $\omega$ in powers of $2-D$ starts at least quadratically as $D<2$.

The exponent is closely related to observables, which are accessible through Monte-Carlo experiments. These are for instance plaquettes-density functions at the repelling potential on a membrane avoiding a single point.

While results for the pinning problem are interesting on its own, the main motivation is certainly to obtain a better understanding of self-avoiding polymerized membranes. Preliminary studies [40] indicate that this problem can also be attacked by the methods developed in this work. This would be very welcome to settle discrepancies between field theoretic results on one side $[17,16,41]$ and numerical results (e.g. [32]) on the other.

\section{A The propagator}

The regularized difference correlator is defined as

$$
C_{a}(x)=G_{a}(0)-G_{a}(x)
$$

where $G_{a}(x)$ denotes the usual two-point correlator, which is obtained from ${ }^{3}$ :

$$
G_{a}(x)=\frac{1}{(2 \pi)^{D}} \int \mathrm{d}^{D} k \frac{\exp \left[i \vec{k} \vec{x}-a^{2} k^{2}\right]}{k^{2}}
$$

\footnotetext{
${ }^{3}$ Strictly speaking, we have to consider the propagator on the torus, as is done in appendix B. However this does not make any difference for the purpose of our argument.
} 
Here, short-wavelength modes are suppressed through a soft cutoff procedure. Introducing a Schwinger parameterization for the evaluation of the integral in (A.2),

$$
\begin{aligned}
G_{a}(x) & =\int_{0}^{\infty} \mathrm{d} t \int \frac{\mathrm{d}^{D} k}{(2 \pi)^{D}} \mathrm{e}^{-\left(t+a^{2}\right) k^{2}} \mathrm{e}^{i k x} \\
& =\frac{1}{(2 \sqrt{\pi})^{D}} \int_{0}^{1 / a^{2}} \mathrm{~d} s s^{D / 2-1} \mathrm{e}^{-s \frac{x^{2}}{4}},
\end{aligned}
$$

where $s=1 /\left(t+a^{2}\right)$, we obtain for (A.1):

$$
C_{a}(x)=\frac{1}{(2 \sqrt{\pi})^{D}} \int_{0}^{1 / a^{2}} \mathrm{~d} s s^{D / 2-1}\left(1-\mathrm{e}^{-s \frac{x^{2}}{4}}\right) .
$$

Further evaluation leads to:

(i) $D=2$ :

$$
C_{a}(x)=\frac{1}{4 \pi}\left(\gamma+\Gamma\left(0, \frac{x^{2}}{4 a^{2}}\right)+\ln \frac{x^{2}}{4 a^{2}}\right) \stackrel{x \rightarrow \infty}{\longrightarrow} \frac{1}{2 \pi} \ln \frac{x}{a}
$$

(ii) $D<2$ :

$$
\begin{aligned}
C_{a}(x) & =\frac{|x|^{2-D} \Gamma\left(\frac{D}{2}\right)}{(2-D) 2 \pi^{D / 2}}+\frac{a^{2-D}}{(2-D) 2^{D-1} \pi^{D / 2}} \mathrm{e}^{-\frac{x^{2}}{4 a^{2}}}-\frac{a^{2-D}}{(2-D) 2^{D-1} \pi^{D / 2}}-\frac{|x|^{2-D} \Gamma\left(\frac{D}{2}, \frac{x^{2}}{4 a^{2}}\right)}{(2-D) 2 \pi^{D / 2}} \\
& \stackrel{\rightarrow \infty}{\simeq} \frac{|x|^{2-D}}{S_{D}(2-D)}-\frac{a^{2-D}}{(2-D) 2^{D-1} \pi^{D / 2}} \stackrel{D \rightarrow 2}{\longrightarrow} \frac{1}{2 \pi} \ln \frac{x}{a} .
\end{aligned}
$$

$\Gamma(z, \alpha)$ denotes the incomplete $\Gamma$-function:

$$
\Gamma(z, \alpha)=\int_{\alpha}^{\infty} \mathrm{d} t t^{z-1} \mathrm{e}^{-t}
$$

Especially:

$$
\lim _{a \rightarrow 0} C_{a}(x)=\frac{|x|^{2-D}}{S_{D}(2-D)}
$$

as long as $D<2$.

\section{B Calculation of the diagrams in the $(2-D)$-expansion}

In this section we calculate the diagrams which appear in the $2-D$ expansion on the torus of size $L=1$. It turns out that to obtain $\overline{\mathbb{C}}$ and $\overline{\mathbb{C}_{c}^{2}}$ we need to evaluate two sums over discrete wave-vectors due to periodic boundary conditions on the torus. Let us first derive the latter before turning to the explicit evaluation. Starting from the definition of the difference correlator $C(x)$,

$$
C(x):=G(x)-G(0)
$$


where $G(x)$ is the usual two-point correlator, we obtain $C(x)$ through an inverse discrete Fourier-transformation from $G(k)=1 / \vec{k}^{2}$, which reads:

$$
C(x)=\sum_{\vec{k} \neq 0} \frac{1}{\vec{k}^{2}}\left(1-\mathrm{e}^{i \vec{k} \vec{x}}\right), \quad \vec{k}=2 \pi \vec{n}, \vec{n} \in \mathbb{Z} \times \mathbb{Z} \backslash\{\overrightarrow{0}\} .
$$

Performing the averaging procedure

$$
\overline{C(x)}=\int_{x} C(x)
$$

where $\int_{x} \mathrm{e}^{i \vec{k} \vec{x}}=\delta_{\vec{k}}^{D}$ is to be taken into account, the calculation of $\overline{C(x)}$ reduces to

$$
\overline{C(x)}=I_{1}:=\sum_{\vec{k} \neq 0} \frac{1}{\vec{k}^{2}}, \quad \vec{k}=2 \pi \vec{n}
$$

where $\vec{k}$ is $D$-dimensional, and the indices $n_{i}$ are integer and running from $-\infty$ to $\infty, \vec{n}=0$ being excluded from the summation. Of course, in the expansion in powers of $2-D$ we need an analytic continuation to real values of $\mathrm{D}$. Finally, to obtain $\overline{\mathbb{C}(x)}$ we have to subtract $\overline{\mathbb{C}^{(0)}(x)}$ from $\overline{C(x)}$. Due to our normalizations:

$$
\overline{\mathbb{C}(x)}=S_{D}\left(\overline{C(x)}-\frac{\overline{\mathbb{C}^{(0)}(x)}}{2 \pi(2-D)}\right),
$$

where $S_{D}$ denotes the volume of the unit sphere and $\overline{\mathbb{C}^{(0)}(x)}=1$.

Turning to $\overline{\mathbb{C}_{c}^{2}(x)}$, we first note that within our normalizations we have

$$
S_{D}^{-2} \overline{\mathbb{C}^{2}(x)}=\overline{\left(C(x)-\mathbb{C}^{(0)}(x) /(2 \pi(2-D))\right)^{2}}=\overline{C(x)^{2}}-2 \frac{\overline{C(x)}}{2 \pi(2-D)}+\frac{1}{(2 \pi(2-D))^{2}}
$$

and

$$
S_{D}^{-2} \overline{\mathbb{C}(x)}^{2}=\overline{C(x)}^{2}-2 \frac{\overline{C(x)}}{2 \pi(2-D)}+\frac{1}{(2 \pi(2-D))^{2}}
$$

according to (B.5), such that

$$
\overline{\mathbb{C}_{c}^{2}(x)} \equiv \overline{\mathbb{C}^{2}(x)}-\overline{\mathbb{C}(x)}^{2}=S_{D}^{2}\left({\overline{C^{2}(x)}}_{\left.-\overline{C(x)}^{2}\right)} .\right.
$$

Knowing already the sum to be evaluated to obtain $\bar{C}$, (B.4), what is left is:

$$
\begin{aligned}
\overline{C^{2}(x)} & =\int_{x} C^{2}(x)=\int_{x} \sum_{\vec{k} \neq 0} \sum_{\vec{p} \neq 0} \frac{1}{\vec{k}^{2}} \frac{1}{\vec{p}^{2}}\left(\mathrm{e}^{i \vec{k} \vec{x}}-1\right)\left(\mathrm{e}^{i \vec{p} \vec{x}}-1\right) \\
& =\sum_{\vec{k} \neq 0} \sum_{\vec{p} \neq 0} \frac{1}{\vec{k}^{2}} \frac{1}{\vec{p}^{2}}\left(\delta_{\vec{k}+\vec{p}}^{D}-\delta_{\vec{k}}^{D}-\delta_{\vec{p}}^{D}+1\right)=\sum_{\vec{k} \neq 0} \frac{1}{\vec{k}^{4}}+\left[\sum_{\vec{k} \neq 0} \frac{1}{\vec{k}^{2}}\right]^{2} .
\end{aligned}
$$


Therefore,

$$
S_{D}^{-2} \overline{\mathbb{C}_{c}^{2}(x)}=I_{2}:=\sum_{\vec{k} \neq 0} \frac{1}{\vec{k}^{4}}, \quad k_{i}=2 \pi n_{i} .
$$

Let us first calculate $I_{1}$ : Introducing a Schwinger parameterization we have:

$$
I_{1}=\frac{1}{(2 \pi)^{2}} \sum_{\substack{n_{i}=-\infty \\ \vec{n} \neq 0}}^{\infty} \frac{1}{\vec{n}^{2}}=\frac{1}{(2 \pi)^{2}} \sum_{\substack{n_{i}=-\infty \\ \vec{n} \neq 0}}^{\infty} \int_{0}^{\infty} \mathrm{d} s \mathrm{e}^{-s \vec{n}^{2}}=\frac{1}{(2 \pi)^{2}} \int_{0}^{\infty} \mathrm{d} s\left[\left(\sum_{n=-\infty}^{\infty} \mathrm{e}^{-s n^{2}}\right)^{D}-1\right]
$$

where it is to be noted that the sum in the last line is only one-dimensional. Furthermore, from now on it is clear, how $I_{1}$ is analytically continued to real values of $D$.

In order to evaluate this sum, we will make use of a Poisson-transformation, which reads:

$$
\sum_{n=-\infty}^{\infty} \mathrm{e}^{-A(n-z / 2)^{2}}=\sqrt{\frac{\pi}{A}} \sum_{l=-\infty}^{\infty} \mathrm{e}^{-\frac{\pi^{2} l^{2}}{A}+i \pi l z}
$$

The contribution from $l=0$ is the approximation of the 1.h.s. through a Gaussian integral. Our aim is to calculate the coefficients of the $2-D$ expansion of $I_{1}$ numerically using some algebraic manipulation program. Then, the integration interval in (B.11) has to be made finite. This is done as follows: For any $s_{0}>0$ we have

$$
\begin{aligned}
I_{1} & =\frac{1}{(2 \pi)^{2}} \int_{0}^{s_{0}^{-1}} \mathrm{~d} s\left[\left(\sum_{n=-\infty}^{\infty} \mathrm{e}^{-s n^{2}}\right)^{D}-1\right]+\frac{1}{(2 \pi)^{2}} \int_{s_{0}^{-1}}^{\infty} \mathrm{d} s\left[\left(\sum_{n=-\infty}^{\infty} \mathrm{e}^{-s n^{2}}\right)^{D}-1\right] \\
& =\frac{1}{(2 \pi)^{2}} \int_{0}^{s_{0}^{-1}} \mathrm{~d} s\left[\left(\sum_{n=-\infty}^{\infty} \mathrm{e}^{-s n^{2}}\right)^{D}-1\right]+\frac{1}{(2 \pi)^{2}} \int_{0}^{s_{0}} \frac{\mathrm{d} s}{s^{2}}\left[\left(\sum_{n=-\infty}^{\infty} \mathrm{e}^{-n^{2} / s}\right)^{D}-1\right] .
\end{aligned}
$$

For any finite $s_{0}>0$, the sum in the r.h.s. integral can be truncated at some finite $n_{\max }$ for all $s \in\left[0, s_{0}\right]$. For the first integral (corresponding to small values of $s$ ) we make use of the poissonian formula (B.12) with $z=0$ :

$$
\sum_{n=-\infty}^{\infty} \mathrm{e}^{-s n^{2}}=\sqrt{\frac{\pi}{s}} \sum_{l=-\infty}^{\infty} \mathrm{e}^{-\pi^{2} l^{2} / s}
$$

Inserting this into (B.13), the sum in the first integral can be truncated at some finite $l$ as well, such that one may approximately write:

$$
I_{1} \approx \frac{1}{(2 \pi)^{2}} \int_{0}^{s_{0}^{-1}} \mathrm{~d} s\left[\left(\sqrt{\frac{\pi}{s}} \sum_{l=-l_{\max }}^{l_{\max }} \mathrm{e}^{-\pi^{2} l^{2} / s}\right)^{D}-1\right]+\frac{1}{(2 \pi)^{2}} \int_{0}^{s_{0}} \frac{\mathrm{d} s}{s^{2}}\left[\left(\sum_{n=-n_{\max }}^{n_{\max }} \mathrm{e}^{-n^{2} / s}\right)^{D}-1\right]
$$


Choosing $s_{0}$ in a way that $l_{\max }$ can be set equal to zero the 1.h.s. integral can be evaluated analytically:

$$
I_{1} \approx \frac{1}{(2 \pi)^{2}}\left(\frac{2 \pi^{D / 2}}{2-D} s_{0}^{D / 2-1}-s_{0}^{-1}\right)+\frac{1}{(2 \pi)^{2}} \int_{0}^{s_{0}} \frac{\mathrm{d} s}{s^{2}}\left[\left(\sum_{n=-n_{\max }}^{n_{\max }} \mathrm{e}^{-n^{2} / s}\right)^{D}-1\right] .
$$

There is a pole in $2-D$, which can be easily subtracted expanding the expression in powers of $2-D$. The pole is

$$
I_{1}=\frac{1}{2 \pi(2-D)}+O\left((2-D)^{0}\right)
$$

The precision of the machine that we used to evaluate (B.16) was sufficient in a way that we could select $s_{0}$ from an interval, such that the sum appearing in the integrand could be truncated at some finite $n_{\max }$ and the result was independent from the precise value of $s_{0}$ within the desired order of accuracy, therefore, justifying the approximation in (B.15). Setting for instance $s_{0}=$ 1.9 and $n_{\max }=20$ we obtain with Mathematica $^{\circledR}$ :

$$
I_{1}=\frac{1}{2 \pi(2-D)}-0.715497(1)-0.00457046(1)(2-D)+O\left((2-D)^{2}\right) .
$$

On the torus we scaled the square root of the volume of the $D$-dimensional unitsphere into the field. Accordingly, comparing with (B.4) and (B.5) we then find:

$$
\overline{\mathbb{C}}=-0.44956(1)+0.3583(1)(2-D)+O\left((2-D)^{2}\right) .
$$

Let us turn to the evaluation of $I_{2}$ following the same strategy as above. Again, setting $L=1$ and introducing a Schwinger parameterization leads to:

$$
\begin{aligned}
I_{2} & =\frac{1}{(2 \pi)^{4}} \int_{0}^{\infty} \mathrm{d} s s\left[\left(\sum_{n=-\infty}^{\infty} \mathrm{e}^{-s n^{2}}\right)^{D}-1\right] \\
& \approx \frac{1}{(2 \pi)^{4}} \int_{0}^{s_{0}^{-1}} \mathrm{~d} s s\left[\left(\sqrt{\frac{\pi}{s}} \sum_{l=-l_{\max }}^{l_{\max }} \mathrm{e}^{-\pi^{2} l^{2} / s}\right)^{D}-1\right]+\frac{1}{(2 \pi)^{4}} \int_{0}^{s_{0}} \frac{\mathrm{d} s}{s^{3}}\left[\left(\sum_{n=-n_{\max }}^{n_{\max }} \mathrm{e}^{-n^{2} / s}\right)^{D}-1\right],
\end{aligned}
$$

where we have once again applied the Poisson-transformation (B.12) with $z=0$ on one part of the integration interval and truncated both series at some finite values $n_{\max }$ and $l_{\max }$.

There is no pole in $2-D$. Since $I_{2}$ appears at second order in $2-D$ we only need its value at $D=2$. $s_{0}$ has to be chosen from an appropriate interval. Setting $n_{\max }=n_{\max }=10$ and $s_{0}=1.1$ we obtain with Mathematica $^{\circledR}$ :

$$
I_{2}=0.00386695(1)+O((2-D)),
$$

or, due to the rescaling by $S_{D}^{2}$,

$$
\overline{\mathbb{C}_{c}^{2}}=0.152661(1)+O((2-D)) .
$$




\section{Acknowledgments}

It is a pleasure to thank R. Blossey, F. David, H.W. Diehl, M. Kardar, and L. Schäfer for useful discussions. We are grateful to Andreas Ludwig for persisting questions, and his never tiring efforts to understand the limit of $D \rightarrow 2$. This work has been supported by the DFG through the Leibniz program Di 378/2-1, under Heisenberg grant Wi 1932/1-1.

\section{R References}

[1] L. Schäfer, Excluded Volume Effects in Polymer Solutions, Springer Verlag, Berlin, Heidelberg, 1999.

[2] J. des Cloizeaux and G. Jannink, Polymers in Solution, Their Modelling and Structure, Clarendon Press, Oxford, 1990.

[3] P.-G. de Gennes, Scaling concepts in polymer physics, Cornell University Press, Ithaca and London, 1979.

[4] E. Eisenriegler, Polymers near surfaces, World Scientific, 1993.

[5] M. Fixman, Excluded volume in polymer chains, J. Chem. Phys. 23 (1955) 1656-1659.

[6] L. Schafer and T.A. Witten, Renormalized field theory of polymer solutions, J. Chem. Phys. 66 (1977) 2121.

[7] J. des Cloizeaux, Polymers in solutions: Principles and applications of a direct renormalization method, J. de Physique 42 (1981) 635-652.

[8] S.F. Edwards, The statistical mechanics of polymers with excluded volume, Proc. Phys. Soc. 85 (1965) 613.

[9] P.-G. De Gennes, Exponents for the excluded volume problem as derived by the Wilson method, Phys. Lett. A 38 (1972) 339-340.

[10] Y. Kantor and D.R. Nelson, Crumpling transition in polymerized membranes, Phys. Rev. Lett. 58 (1987) 2774-2777.

[11] Y. Kantor and D.R. Nelson, Phase transitions in flexible polymeric surfaces, Phys. Rev. A 36 (1987) 4020-4032.

[12] Y. Kantor, M. Kardar and D.R. Nelson, Statistical mechanics of tethered surfaces, Phys. Rev. Lett. 57 (1986) 791-795.

[13] Y. Kantor, M. Kardar and D.R. Nelson, Tethered surfaces: Statics and dynamics, Phys. Rev. A 35 (1987) 3056-3071.

[14] M. Paczuski, M. Kardar and D.R. Nelson, Landau theory of the crumpling transition, Phys. Rev. Lett. 60 (1988) 2638. 
[15] M. Paczuski and M. Kardar, Renormalization-group analysis of the crumpling transition in large d, Phys. Rev. A 39 (1989) 6086-6089.

[16] F. David and K.J. Wiese, Scaling of self-avoiding tethered membranes: 2-loop renormalization group results, Phys. Rev. Lett. 76 (1996) 4564.

[17] K.J. Wiese and F. David, New renormalization group results for scaling of self-avoiding tethered membranes, Nucl. Phys. B 487 (1997) 529-632.

[18] K.J. Wiese, Polymerized membranes, a review. Volume 19 of Phase Transitions and Critical Phenomena, Acadamic Press, London, 1999.

[19] M. Kardar and D.R. Nelson, $\varepsilon$ expansions for crumpled manifolds, Phys. Rev. Lett. 58 (1987) 1289 and 2280 E.

[20] J.A. Aronovitz and T.C. Lubensky, Fluctuations of solid membranes, Phys. Rev. Lett. 60 (1988) 2634-2637.

[21] F. David, B. Duplantier and E. Guitter, Renormalization and hyperscaling for selfavoiding manifold models, Phys. Rev. Lett. 72 (1994) 311.

[22] F. David, B. Duplantier and E. Guitter, Renormalization theory for the self-avoiding polymerized membranes, cond-mat/9702136 (1997).

[23] T. Hwa, Generalized $\varepsilon$ expansion for self-avoiding tethered manifolds, Phys. Rev. A 41 (1990) 1751-1756.

[24] K.J. Wiese and F. David, Self-avoiding tethered membranes at the tricritical point, Nucl. Phys. B 450 (1995) 495-557.

[25] R.R. Chianelli, E.B. Prestridge, T.A. Pecorado and J.P. de Neufville, Molybdenum disulfide in the poorly crystalline "rag” structure, Science 203 (1979) 1105.

[26] T. Hwa, E. Kokufuta and T. Tanaka, Conformation of graphite oxide membranes in solution, Phys. Rev. A 44 (1991) 2235.

[27] X. Wen, C.W. Garland, T. Hwa, M. Kardar, E. Kokufuta, Y. Li, M. Orkisz and T. Tanaka, Crumpled and collapsed conformations in graphite oxide membranes, Nature 355 (1992) 426.

[28] M.S. Spector, E. Naranjo, S. Chiruvolu and J.A. Zasadzinski, Conformations of a tethered membrane: Crumpling in graphitic oxide?, Phys. Rev. Lett. 73 (1994) 2867-2870.

[29] A. Baumgärtner, Does a polymerized membrane crumple?, J. Phys. I France 1 (1991) 1549-1556.

[30] A. Baumgärtner and W. Renz, Crumpled self-avoiding tethered surfaces, Europhys. Lett. 17 (1992) 381-386.

[31] D.M. Kroll and G. Gompper, Floppy tethered networks, J. Phys. I France 3 (1993) 1131. 
[32] G. Thorleifsson M. Bowick, A. Cacciuto and A. Travesset, Universality classes of selfavoiding fixed-connectivity membranes, Eur. Phys. J. E 5 (2001) 149.

[33] H.A. Pinnow and K.J. Wiese, Interacting crumpled manifolds, J. Phys. A 35 (2002) 1195-1229.

[34] H.A. Pinnow and K.J. Wiese, Interacting crumpled manifolds: Exact results to all orders of perturbation theory, Europhys. Lett. 64 (2003) 371-377.

[35] F. David, B. Duplantier and E. Guitter, Renormalization of crumpled manifolds, Phys. Rev. Lett. 70 (1993) 2205.

[36] F. David, B. Duplantier and E. Guitter, Renormalization theory for interacting crumpled manifolds, Nucl. Phys. B 394 (1993) 555-664.

[37] G. Forgas, R. Lipowsky and T.M. Nieuwenhuizen, The behaviour of interfaces in ordered and disordered systems. Volume 14 of Phase Transitions and Critical Phenomena, pages 136-376, Academic Press London, 1991.

[38] E. Brézin, B.I. Halperin and S. Leibler, Critical wetting in three dimensions, Phys. Rev. Lett. 50 (1983) 1387.

[39] P.J. Upton, Exact interface model for wetting in the planar ising model, Phys. Rev. E 60 (1999) 3475-3478.

[40] H.A. Pinnow and K.J. Wiese, work in progress.

[41] F. David and K.J. Wiese, Large orders for self-avoiding membranes, Nucl. Phys. B 535 (1998) 555-595. 